\title{
The IceProd Framework: Distributed Data Processing for the IceCube Neutrino Observatory
}

M. G. Aartsen ${ }^{\mathrm{b}}$, R. Abbasi ${ }^{\mathrm{ac}}$, M. Ackermann ${ }^{\mathrm{at}}$, J. Adams ${ }^{\mathrm{o}}$, J. A. Aguilar ${ }^{\mathrm{w}}$, M. Ahlers ${ }^{\mathrm{ac}}$, D. Altmann ${ }^{\mathrm{v}}$, C. Arguelles ${ }^{\mathrm{ac}}$, J. Auffenberg ${ }^{\text {ac }}$, X. Bai ${ }^{\text {ah, }, 1}$, M. Baker ${ }^{\text {ac }}$, S. W. Barwick ${ }^{\mathrm{y}}$, V. Baum ${ }^{\mathrm{ae}}$, R. Bay ${ }^{\mathrm{g}}$, J. J. Beatty ${ }^{\mathrm{q}, \mathrm{r}}$, J. Becker Tjus ${ }^{\mathrm{j}}$, K.-H. Becker ${ }^{\text {as }}$, S. BenZvi ${ }^{\text {ac }}$, P. Berghaus ${ }^{\text {at }}$, D. Berley ${ }^{\mathrm{p}}$, E. Bernardini ${ }^{\mathrm{at}}$, A. Bernhard ${ }^{\mathrm{ag}}$, D. Z. Besson ${ }^{\mathrm{aa}}$, G. Binder ${ }^{\mathrm{h}, \mathrm{g}}$, D. Bindigas, M. Bissok ${ }^{\mathrm{a}}$, E. Blaufuss ${ }^{\mathrm{p}}$, J. Blumenthal ${ }^{\mathrm{a}}$, D. J. Boersma ${ }^{\text {ar }}$, C. Bohm ${ }^{\text {ak }}$, D. Bose ${ }^{\mathrm{am}}$, S. Böser ${ }^{\mathrm{k}}$, O. Botner ${ }^{\text {ar }}$, L. Brayeur ${ }^{\mathrm{m}}$, H.-P. Bretz ${ }^{\text {at }}$, A. M. Brown ${ }^{\mathrm{o}}$, R. Bruijn ${ }^{\mathrm{z}}$, J. Casey ${ }^{\mathrm{e}}$, M. Casier ${ }^{\mathrm{m}}$, D. Chirkin ${ }^{\text {ac }}$, A. Christov ${ }^{\mathrm{w}}$, B. Christy ${ }^{\mathrm{p}}$, K. Clark ${ }^{\text {an }}$, L. Classen ${ }^{\mathrm{v}}$, F. Clevermann ${ }^{\mathrm{t}}$, S. Coenders ${ }^{\mathrm{a}}$, S. Cohen ${ }^{\mathrm{z}}$, D. F. Cowen ${ }^{\text {aq,ap }}$, A. H. Cruz Silva ${ }^{\text {at }}$, M. Danninger ${ }^{\mathrm{ak}}$, J. Daughhetee ${ }^{\mathrm{e}}$, J. C. Davis ${ }^{\mathrm{q}}$, M. Day ${ }^{\mathrm{ac}}$, C. De Clercq $^{\mathrm{m}}$, S. De Ridder ${ }^{\mathrm{x}}$, P. Desiatiac,*,

K. D. de Vries ${ }^{\mathrm{m}}$, M. de With ${ }^{\mathrm{i}}$, T. DeYoung ${ }^{\text {aq }}$, J. C. Díaz-Vélez ${ }^{\mathrm{ac}, * *}$, M. Dunkman ${ }^{\mathrm{aq}}$, R. Eagan ${ }^{\text {aq }}$, B. Eberhardt ${ }^{\text {ae }}$, B. Eichmann ${ }^{\mathrm{j}}$, J. Eisch ${ }^{\text {ac }}$, S. Euler ${ }^{\mathrm{a}}$, P. A. Evenson ${ }^{\text {ah }}$, O. Fadiran ${ }^{\text {ac, }}$, A. R. Fazely ${ }^{\mathrm{f}}$, A. Fedynitch ${ }^{\mathrm{j}}$, J. Feintzeig ${ }^{\text {ac }}$, T. Feusels ${ }^{\mathrm{x}}$, K. Filimonov ${ }^{\mathrm{g}}$, C. Finley ${ }^{\mathrm{ak}}$, T. Fischer-Wasels ${ }^{\mathrm{as}}$, S. Flis ${ }^{\mathrm{ak}}$, A. Franckowiak ${ }^{\mathrm{k}}$, K. Frantzen ${ }^{\mathrm{t}}$, T. Fuchs ${ }^{\mathrm{t}}$, T. K. Gaisser ${ }^{\text {ah }}$, J. Gallagher ${ }^{\text {ab }}$, L. Gerhardt ${ }^{\mathrm{h}, \mathrm{g}}$, L. Gladstone ${ }^{\text {ac }}$, T. Glüsenkamp ${ }^{\text {at }}$, A. Goldschmidt ${ }^{\mathrm{h}}$, G. Golup ${ }^{\mathrm{m}}$,

J. G. Gonzalez ${ }^{\text {ah }}$, J. A. Goodman ${ }^{\text {, }, ~ D . ~ G o ́ r a ~}{ }^{\mathrm{v}}$, D. T. Grandmont ${ }^{\mathrm{u}}$, D. Grant ${ }^{\mathrm{u}}$, P. Gretskov ${ }^{\mathrm{a}}$, J. C. Groh ${ }^{\mathrm{aq}}$, A. Groß ${ }^{\mathrm{ag}}$, C. Ha ${ }^{\mathrm{h}, \mathrm{g}}$, A. Haj Ismail ${ }^{\mathrm{x}}$, P. Hallen ${ }^{\mathrm{a}}$, A. Hallgren ${ }^{\mathrm{ar}}$, F. Halzen ${ }^{\mathrm{ac}}$, K. Hanson ${ }^{1}$, D. Hebecker ${ }^{\mathrm{k}}$, D. Heereman ${ }^{1}$,

D. Heinen ${ }^{\mathrm{a}}$, K. Helbing ${ }^{\text {as }}$, R. Hellauer ${ }^{\mathrm{p}}$, S. Hickford ${ }^{\mathrm{o}}$, G. C. Hill ${ }^{\mathrm{b}}$, K. D. Hoffman ${ }^{\mathrm{p}}$, R. Hoffmann ${ }^{\text {as }}$, A. Homeier ${ }^{\mathrm{k}}$,

K. Hoshina ${ }^{\text {ac }}$, F. Huang aq, W. Huelsnitz ${ }^{\text {p }}$ P. O. Hulth ${ }^{\text {ak }}$, K. Hultqvist ${ }^{\text {ak }}$, S. Hussain ${ }^{\text {ah }}$, A. Ishihara ${ }^{\text {n}}$, E. Jacobi ${ }^{\text {at }}$,

J. Jacobsen ${ }^{\text {ac }}$, K. Jagielski ${ }^{\text {a }}$, G. S. Japaridze ${ }^{\text {d }}$, K. Jero ${ }^{\text {ac }}$, O. Jlelati ${ }^{\mathrm{x}}$, B. Kaminsky ${ }^{\text {at }}$, A. Kappes ${ }^{\mathrm{v}}$, T. Karg ${ }^{\text {at }}$, A. Karle ${ }^{\text {ac }}$,

M. Kauerac, J. L. Kelley ac, J. Kiryluk ${ }^{\text {al }}$, J. Kläs ${ }^{\text {as }}$, S. R. Klein ${ }^{\text {h,g }}$, J.-H. Köhne ${ }^{\text {t }}$, G. Kohnen ${ }^{\text {af }}$, H. Kolanoski ${ }^{i}$,

L. Köpke ${ }^{\text {ae }}$, C. Kopperac, S. Kopper ${ }^{\text {as }}$, D. J. Koskinen ${ }^{\mathrm{s}}$, M. Kowalski ${ }^{\mathrm{k}}$, M. Krasberg ${ }^{\text {ac }}$, A. Kriesten ${ }^{\mathrm{a}}$, K. Krings ${ }^{\mathrm{a}}$, G. Kroll ${ }^{\text {ae }}$, J. Kunnen ${ }^{\mathrm{m}}$, N. Kurahashic ${ }^{\text {ac }}$, T. Kuwabara ${ }^{\text {ah }}$, M. Labare ${ }^{\mathrm{x}}$, H. Landsman ${ }^{\text {ac }}$, M. J. Larson ${ }^{\text {ao }}$, M. Lesiak-Bzdak ${ }^{\mathrm{al}}$, M. Leuermann ${ }^{\mathrm{a}}$, J. Leute ${ }^{\mathrm{ag}}$, J. Lünemann ${ }^{\text {ae }}$, O. Macías ${ }^{\mathrm{o}}$, J. Madsen ${ }^{\mathrm{aj}}$, G. Maggi ${ }^{\mathrm{m}}$,

R. Maruyama ${ }^{\text {ac }}$, K. Mase ${ }^{\text {n}}$, H. S. Matis ${ }^{\text {h }}$, F. McNally ${ }^{\text {ac }}$, K. Meagher ${ }^{\mathrm{p}}$, M. Merck ${ }^{\text {ac }}$, G. Merino ${ }^{\text {ac }}$, T. Meures ${ }^{1}$, S. Miarecki ${ }^{\text {h,g }}$, E. Middell ${ }^{\text {at }}$, N. Milke ${ }^{\mathrm{t}}$, J. Miller ${ }^{\mathrm{m}}$, L. Mohrmann ${ }^{\text {at }}$, T. Montaruli ${ }^{\mathrm{w}, 2}$, R. Morse ${ }^{\mathrm{ac}}$, R. Nahnhauer ${ }^{\text {at }}$,

U. Naumann ${ }^{\text {as }}$, H. Niederhausen al , S. C. Nowicki ${ }^{\mathrm{u}}$, D. R. Nygren ${ }^{\mathrm{h}}$, A. Obertacke ${ }^{\text {as }}$, S. Odrowski ${ }^{\mathrm{u}}$, A. Olivas ${ }^{\mathrm{p}}$,

A. Omairat ${ }^{\mathrm{as}}$, A. O’Murchadha ${ }^{\mathrm{l}}$, L. Paul ${ }^{\mathrm{a}}$, J. A. Pepper ${ }^{\mathrm{a}}$, C. Pérez de los Heros ${ }^{\mathrm{ar}}$, C. Pfendner ${ }^{\mathrm{q}}$, D. Pieloth ${ }^{\mathrm{t}}$, E. Pinat ${ }^{\mathrm{l}}$,

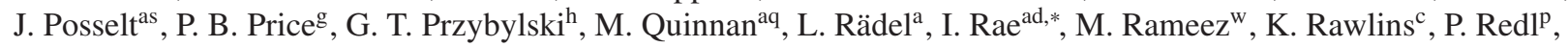

R. Reimann ${ }^{\mathrm{a}}$, E. Resconia ${ }^{\mathrm{ag}}$, W. Rhode ${ }^{\mathrm{t}}$, M. Ribordy ${ }^{\mathrm{z}}$, M. Richman ${ }^{\mathrm{p}}$, B. Riedel ${ }^{\mathrm{ac}}$, J. P. Rodrigues ${ }^{\mathrm{ac}}$, C. Rott $^{\mathrm{am}}$,

T. Ruhe ${ }^{\mathrm{t}}$, B. Ruzybayev ${ }^{\text {ah }}$, D. Ryckbosch ${ }^{\mathrm{x}}$, S. M. Saba ${ }^{\mathrm{j}}$, H.-G. Sander ${ }^{\mathrm{ae}}$, M. Santander ${ }^{\mathrm{ac}}$, S. Sarkar ${ }^{\mathrm{s}, \mathrm{ai}}$, K. Schatto ${ }^{\mathrm{ae}}$,

F. Scheriau ${ }^{t}$, T. Schmidt ${ }^{\mathrm{p}}$, M. Schmitz ${ }^{\mathrm{t}}$, S. Schoenen ${ }^{\mathrm{a}}$, S. Schöneberg ${ }^{\mathrm{j}}$, A. Schönwald ${ }^{\text {at }}$, A. Schukraft ${ }^{\mathrm{a}}$, L. Schulte ${ }^{\mathrm{k}}$,

D. Schultz ${ }^{\text {ac,*, O. Schulz }}{ }^{\mathrm{ag}}$, D. Seckel ${ }^{\mathrm{ah}}$, Y. Sestayo $^{\mathrm{ag}}$, S. Seunarine ${ }^{\mathrm{aj}}$, R. Shanidze ${ }^{\text {at }}$, C. Sheremata ${ }^{\mathrm{u}}$, M. W. E. Smith ${ }^{\text {aq }}$, D. Soldin ${ }^{\text {as }}$, G. M. Spiczak ${ }^{\text {aj }}$, C. Spiering ${ }^{\text {at }}$, M. Stamatikos ${ }^{\text {q,3 }}$, T. Stanev ${ }^{\text {ah }}$, N. A. Stanisha ${ }^{\text {aq }}$, A. Stasik ${ }^{\mathrm{k}}$,

T. Stezelberger ${ }^{\text {h }}$, R. G. Stokstad ${ }^{\mathrm{h}}$, A. Stöß1 ${ }^{\text {at }}$, E. A. Strahler ${ }^{\mathrm{m}}$, R. Ström ${ }^{\text {ar }}$, N. L. Strotjohann ${ }^{\mathrm{k}}$, G. W. Sullivan ${ }^{\mathrm{p}}$,

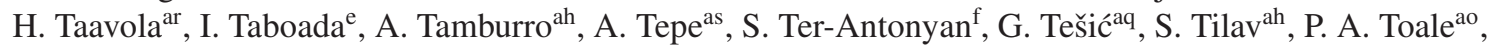

M. N. Tobin ${ }^{\text {ac }}$, S. Toscano ${ }^{\text {ac }}$, M. Tselengidou ${ }^{\mathrm{v}}$, E. Unger ${ }^{\mathrm{j}}$, M. Usner ${ }^{\mathrm{k}}$, S. Vallecorsa ${ }^{\mathrm{w}}$, N. van Eijndhoven ${ }^{\mathrm{m}}$,

A. Van Overloop ${ }^{\mathrm{x}}$, J. van Santen ${ }^{\mathrm{ac}}$, M. Vehring ${ }^{\mathrm{a}}$, M. Voge ${ }^{\mathrm{k}}$, M. Vraeghe ${ }^{\mathrm{x}}$, C. Walck $^{\mathrm{ak}}$, T. Waldenmaier ${ }^{\mathrm{i}}$, M. Wallraff ${ }^{\mathrm{a}}$,

Ch. Weaver ${ }^{\text {ac }}$, M. Wellons ${ }^{\text {ac }}$, C. Wendt ${ }^{\text {ac }}$, S. Westerhoff ${ }^{\text {ac }}$, N. Whitehorn ${ }^{\text {ac }}$, K. Wiebe ${ }^{\text {ae }}$, C. H. Wiebusch ${ }^{\mathrm{a}}$,

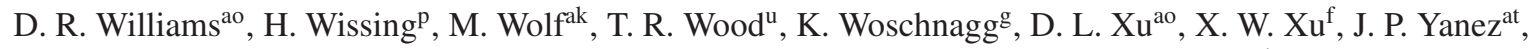
G. Yodh ${ }^{\mathrm{y}}$, S. Yoshida ${ }^{\mathrm{n}}$, P. Zarzhitsky ${ }^{\text {ao }}$, J. Ziemann ${ }^{\mathrm{t}}$, S. Zierke ${ }^{\mathrm{a}}$, M. Zoll ${ }^{\mathrm{ak}}$

${ }^{a}$ III. Physikalisches Institut, RWTH Aachen University, D-52056 Aachen, Germany
${ }^{b}$ School of Chemistry E Physics, University of Adelaide, Adelaide SA, 5005 Australia

\footnotetext{
${ }^{*}$ Corresponding author

** Principal corresponding author

Email addresses: desiati@icecube.wisc.edu (P. Desiati), juancarlos@wipac.wisc.edu (J. C. Díaz-Vélez), of adiran@icecube.wisc.edu (O. Fadiran), ian@cs.wisc.edu (I. Rae), dschultz@icecube.wisc.edu (D. Schultz)

${ }^{1}$ Physics Department, South Dakota School of Mines and Technology, Rapid City, SD 57701, USA

2 also Sezione INFN, Dipartimento di Fisica, I-70126, Bari, Italy

${ }^{3}$ NASA Goddard Space Flight Center, Greenbelt, MD 20771, USA

Preprint submitted to Journal of Parallel and Distributed Computing
} 


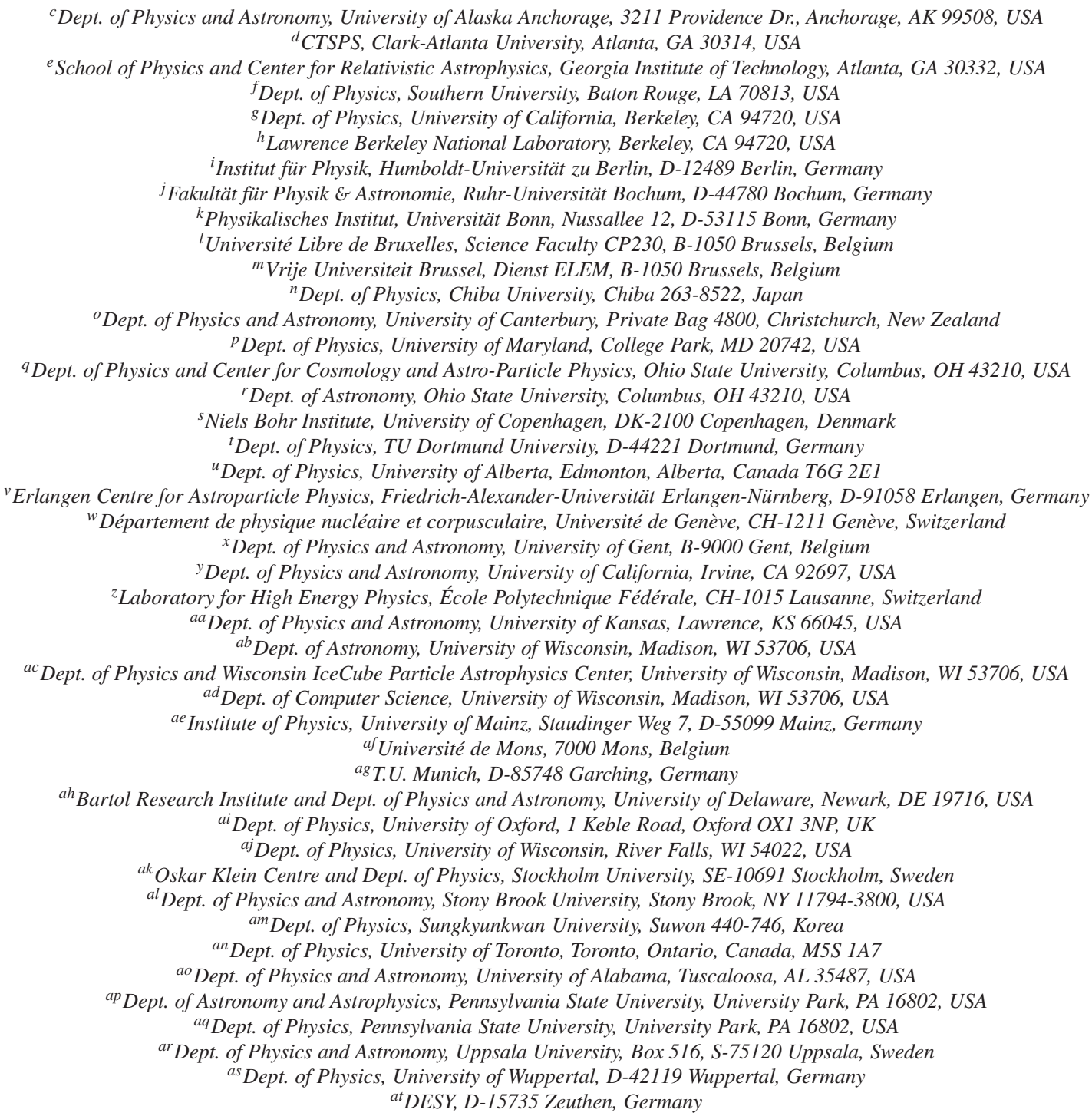

\begin{abstract}
IceCube is a one-gigaton instrument located at the geographic South Pole, designed to detect cosmic neutrinos, identify the particle nature of dark matter, and study high-energy neutrinos themselves. Simulation of the IceCube detector and processing of data require a significant amount of computational resources. This paper presents the first detailed description of IceProd, a lightweight distributed management system designed to meet these requirements. It is driven by a central database in order to manage mass production of simulations and analysis of data produced by the IceCube detector. IceProd runs as a separate layer on top of other middleware and can take advantage of a variety of computing resources, including grids and batch systems such as CREAM, HTCondor, and PBS. This is accomplished by a set of dedicated daemons that process job submission in a coordinated fashion through the use of middleware plugins that serve to abstract the details of job submission and job management from the framework.
\end{abstract}

Key words: Data Management, Grid Computing, Monitoring, Distributed Computing 


\section{Introduction}

Large experimental collaborations often need to produce extensive volumes of computationally intensive Monte Carlo simulations and process vast amounts of data. These tasks are usually farmed out to large computing clusters or grids. For such large datasets, it is important to be able to document details associated with each task, such as software versions and parameters like the pseudo-random number generator seeds used for each dataset. Individual members of such collaborations might have access to modest computational resources that need to be coordinated for production. Such computational resources could also potentially be pooled in order to provide a single, more powerful, and more productive system that can be used by the entire collaboration. This article describes the design of a software package meant to address all of these concerns. It provides a simple way to coordinate processing and storage of large datasets by integrating grids and small clusters.

\subsection{The IceCube Detector}

The IceCube detector shown in Figure 1 is located at the geographic South Pole and was completed at the end of 2010 [1,2]. It consists of 5160 optical sensors buried between 1450 and 2450 meters below the surface of the South Pole ice sheet and is designed to detect interactions of neutrinos of astrophysical origin [1]. However, it is also sensitive to downward-going highly energetic muons and neutrinos produced in cosmic-rayinduced air showers. IceCube records $\sim 10^{10}$ cosmic-ray events per year. The cosmic-ray-induced muons outnumber neutrino-induced events (including ones from atmospheric origin) by about 500,000:1. They represent a background for most IceCube analyses and are filtered prior to transfer to the data processing center in the Northern Hemisphere. Filtering at the data collection source is required because of bandwidth limitations on the satellite connection between the detector and the processing location [3]. About $100 \mathrm{~GB}$ of data from the IceCube detector is transferred to the main data storage facility daily. In order to facilitate record keeping, the data is divided into runs, and each run is further subdivided into multiple files. The size of each file is dictated by what is considered optimal for storage and access. Each run typically consists of hundreds of files, resulting in $\sim 400,000$ files for each year of detector operation. Once the data has been transferred, additional, more computationally-intensive event reconstructions are performed and the data is filtered to select events for various analyses. The computing requirements for the various levels of data processing are shown in Table 1. In order to develop event reconstructions, perform analyses, and understand systematic uncertainties, physicists require statistics from Monte Carlo simulations that are comparable to the data collected by the detector. This requires thousands of years of CPU processing time as can be seen from Table 2 .

Table 1: Data processing demands. Data is filtered on 400 cores at the South Pole using loose selection criteria to reduce volume by a factor of 10 before satellite transfer to the Northern Hemisphere (Level1). Once in the North, more computationally intensive event reconstructions are performed in order to further reduce background contamination (Level2). Further event selections are made for each analysis channel (Level3). Each run is equivalent to approximately eight hours of detector livetime and the processing time is based on a $2.8 \mathrm{GHz}$ core.

\begin{tabular}{l|r|r}
\hline \hline Filter & Processing time/run & Total per year \\
\hline Level1 & $2400 \mathrm{~h}$ & $2.6 \times 10^{6} \mathrm{~h}$ \\
Level2 & $9500 \mathrm{~h}$ & $1.0 \times 10^{7} \mathrm{~h}$ \\
Level3 & $15 \mathrm{~h}$ & $1.6 \times 10^{4} \mathrm{~h}$ \\
\hline
\end{tabular}

Table 2: Runtime of various Monte Carlo simulations of background cosmic-ray shower events and neutrino signal with different energy distributions. The median energy is based on the distribution of events that trigger the detector. The number of events reflects the typical peryear requirements for IceCube analyses.

\begin{tabular}{l|l|r|l}
\hline \hline Simulation & Med. Energy & t/event & events \\
\hline Air showers & $1.2 \times 10^{4} \mathrm{GeV}$ & $5 \mathrm{~ms}$ & $\sim 10^{14}$ \\
Neutrinos & $3.9 \times 10^{6} \mathrm{GeV}$ & $316 \mathrm{~ms}$ & $\sim 10^{8}$ \\
Neutrinos & $8.1 \times 10^{1} \mathrm{GeV}$ & $53 \mathrm{~ms}$ & $\sim 10^{9}$ \\
\hline
\end{tabular}

\subsection{IceCube Computing Resources}

The IceCube collaboration is comprised of 43 research institutions from Europe, North America, Japan, Australia, and New Zealand. Members of the collaboration have access to 25 different computing clusters and grids in Europe, Japan, Canada and the U.S. These range from small computer farms of 30 nodes to large grids, such as the European Grid Infrastructure (EGI), Swedish Grid Initiative (SweGrid), Canada's WestGrid and the Open Science Grid (OSG), that may each have thousands of computing nodes. The total number of nodes available to IceCube member institutions varies with time since much of our use is opportunistic and availability depends on the usage by other projects and experiments. In total, IceCube simulation has run on more than 11,000 distinct multicore computing nodes.

\footnotetext{
${ }^{1} 1 \mathrm{GeV}=10^{9}$ electronvolts (unit of energy)
} 


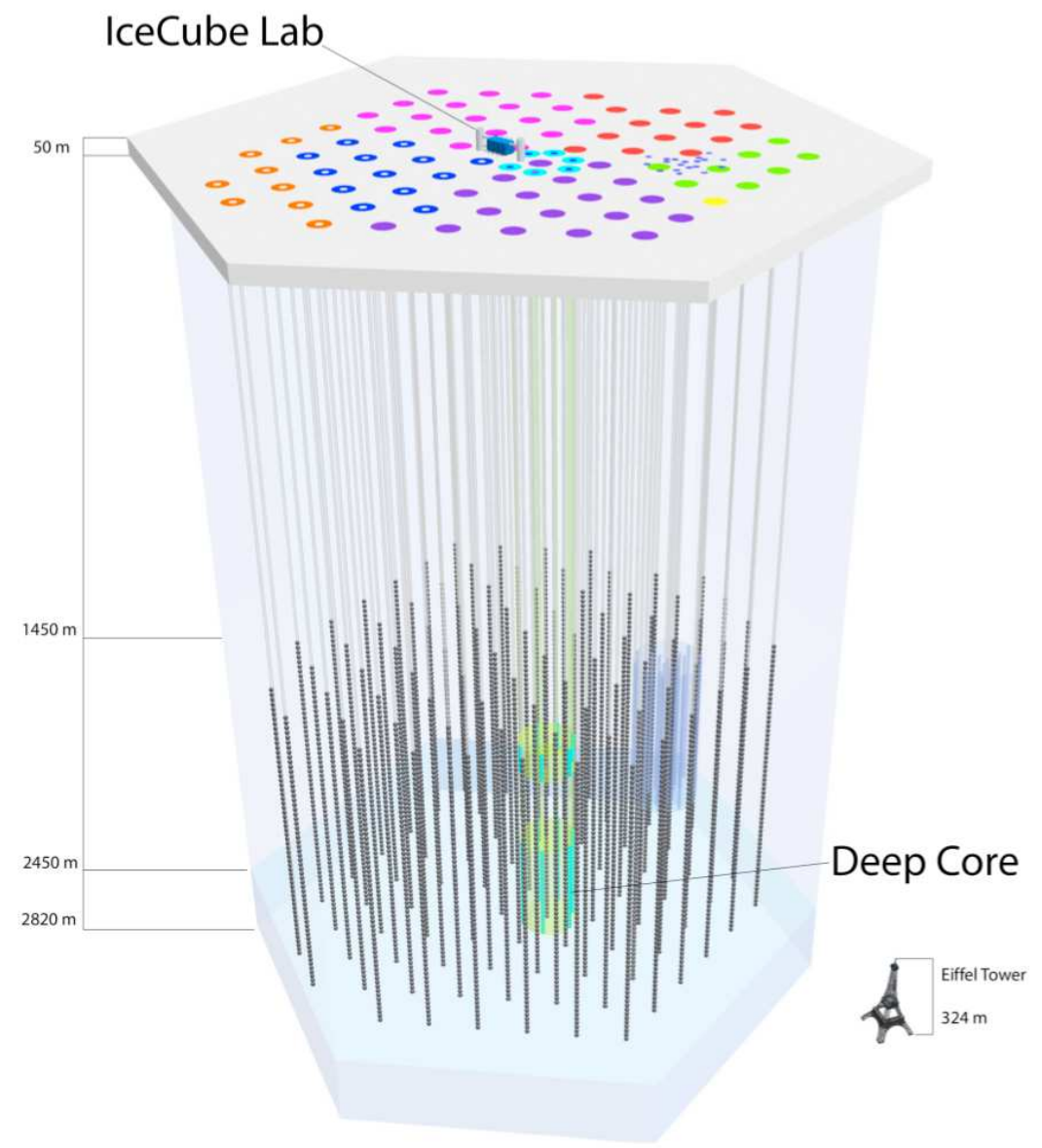

Figure 1: The IceCube detector: the dotted lines at the bottom represent the instrumented portion of the ice. The circles on the top surface represent IceTop, a surface air-shower subdetector. 
On average, IceCube simulation production has run concurrently on $\sim 4,000$ cores at any given time since deployment, and it is anticipated to run on $\sim 5,000$ cores simultaneously during upcoming productions.

\section{IceProd}

The IceProd framework is a software package developed for IceCube with the goal of managing productions across distributed systems and pooling together isolated computing resources that are scattered across member institutions of the Collaboration and beyond. It consists of a central database and a set of daemons that are responsible for the management of grid jobs and data handling through the use of existing grid technology and network protocols.

IceProd makes job scripting easier and sharing productions more efficient. In many ways it is similar to PANDA Grid, the analysis framework for the PANDA experiment [4], in that both tools are distributed systems based on a central database and an interface to local batch systems. Unlike PANDA Grid which depends heavily on AliEn, the grid middleware for the ALICE experiment [5], and on the ROOT analysis framework [6], IceProd was built in-house with minimal software requirements and is not dependent on any particular middleware or analysis framework. It is designed to run completely in user space with no administrative access, allowing greater flexibility in installation. IceProd also includes a built-in monitoring system with no dependencies on any external tools for this purpose. These properties make IceProd a very lightweight yet powerful tool and give it a greater scope beyond IceCube-specific applications.

The software package includes a set of libraries, executables and daemons that communicate with the central database and coordinate to share responsibility for the completion of tasks. The details of job submission and management in different grid environments are abstracted through the use of plugin modules that will be discussed in Section 3.2.1.

IceProd can be used to integrate an arbitrary number of sites including clusters and grids. It is, however, not a replacement for other cluster and grid management tools or any other middleware. Instead, it runs on top of these as a separate layer providing additional functionality. IceProd fills a gap between the user or production manager and the powerful middleware and batch system tools available on computing clusters and grids.

Many of the existing middleware tools, including Condor-C, Globus and CREAM, make it possible to interface any number of computing clusters into a larger pool. However, most of these tools need to be installed and configured by system administrators and, in some cases, customization for general purpose applications is not feasible. In contrast to most of these applications, IceProd runs at the user level and does not require administrator privileges. This makes it possible for individual users to build large production systems by pooling small computational resources together.

Security and data integrity are concerns in any software architecture that depends heavily on communication through the Internet. IceProd includes features aimed at minimizing security and data corruption risks. Security and data integrity are addressed in Section 3.8.

The IceProd client provides a graphical user interface (GUI) for configuring simulations and submitting jobs through a "production server." It provides a method for recording all the software versions, physics parameters, system settings, and other steering parameters associated with a job in a central production database. IceProd also includes a web interface for visualization and live monitoring of datasets. Details about the GUI client and a text-based client are discussed in Section 3.5.

\section{Design Elements of IceProd}

The IceProd software package can be logically divided into the following components or software libraries:

- iceprod-core-a set of modules and libraries of common use throughout IceProd.

- iceprod-server - a collection of daemons and libraries to manage and schedule job submission and monitoring.

- iceprod-modules-a collection of predefined classes that provide an interface between IceProd and an arbitrary task to be performed on a computing node, as defined in Section 3.3.

- iceprod-client - a client (both graphical and text) that can download, edit, and submit dataset steering files to be processed.

- A database that stores configured parameters, libraries (including version information), job information, and performance statistics.

- A web application for monitoring and controlling dataset processing.

These components are described in further detail in the following sections. 


\subsection{IceProd Core Package}

The iceprod-core package contains modules and libraries common to all other IceProd packages. These include classes and methods for writing and parsing XML files and transporting data. The classes that define job execution on a host are contained in this package. The iceprod-core also includes an interpreter (Section 3.1.3) for a simple scripting language that provides some flexibility for parsing XML steering files.

\subsubsection{The JEP}

One of the complications of operating on heterogeneous systems is the diversity of architectures, operating systems, and compilers. IceProd uses HTCondor's NMI-Metronome build and test system [7] for building the IceCube software on a variety of platforms and storing the built packages on a server. As part of the management of each job, IceProd submits a Job Execution Pilot (JEP) to the cluster/grid queue. This script determines what platform a job is running on and, after contacting the monitoring server, which software package to download and execute. During runtime, the JEP performs status updates through the monitoring server via remote procedure calls using XML-RPC [8]. This information is updated on the database and is displayed on the monitoring web interface. Upon completion, the JEP removes temporary files and directories created for the job. Depending on the configuration, it will also cache a copy of the software used, making it available for future JEPs. When caching is enabled, an MD5 checksum is performed on the cached software and compared to what is stored on the server in order to avoid using corrupted or outdated software.

Jobs can fail under many circumstances. These failures include failed submissions due to transient system problems and execution failures due to problems with the execution host. At a higher level, errors specific to IceProd include communication problems with the monitoring daemon or the data repository. In order to account for possible transient errors, the design of IceProd includes a set of states through which a job will transition in order to guarantee successful completion of a well-configured job. The state diagram for an IceProd job is depicted in Figure 2.

\subsubsection{XML Job Description}

In the context of this document, a dataset is defined as a collection of jobs that share a basic set of scripts and software but whose input parameters depend on the ID of each individual job. A configuration or steering file describes the tasks to be executed for an entire dataset.

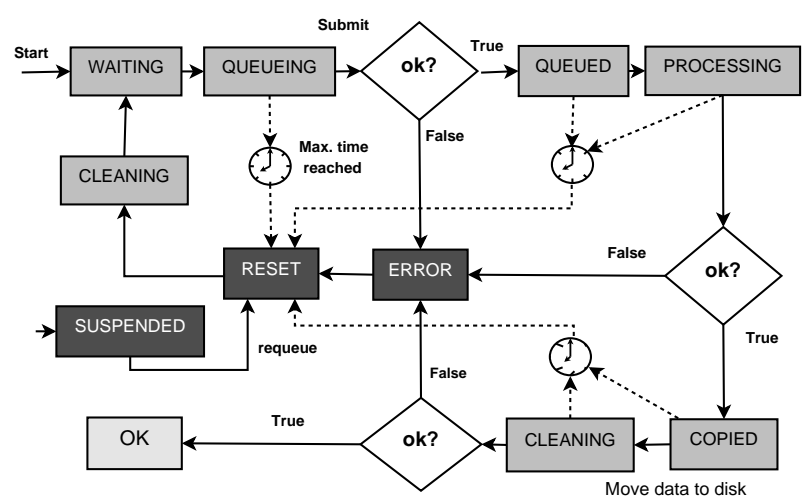

Figure 2: State diagram for the JEP. Each of the nonerror states through which a job passes includes a configurable timeout. The purpose of this timeout is to account for any communication errors that may have prevented a job from setting its status correctly.

IceProd steering files are XML documents with a defined schema. These steering files include information about the specific software versions used for each of the sections, known as trays (a term borrowed from IceTray, the $\mathrm{C}++$ software framework used by the IceCube Collaboration [9]). An IceProd tray represents an instance of an environment corresponding to a set of libraries and executables and a chain of configurable modules with corresponding parameters and input files needed for the job. In addition, there is a header section for user-defined parameters and expressions that are globally accessible by different modules.

\subsubsection{IceProd XML expressions}

A limited programming language was developed in order to allow more scripting flexibility that depends on runtime parameters such as job ID, run ID, and dataset ID. This lightweight, embedded, domain-specific language (DSL) allows for a single XML job description to be applied to an entire dataset following an SPMD (single process, multiple data) paradigm. It is powerful enough to give some flexibility but sufficiently restrictive to limit abuse. Examples of valid expressions include the following:

- $\$ \operatorname{args}(\langle v a r\rangle)$-a command line argument passed to the job (such as job ID or dataset ID).

- \$steering (<var >) —a user defined variable.

- \$system(<var〉) -a system-specific parameter defined by the server.

- \$eval (<expr >) - a mathematical or logical expression (in Python). 
- \$sprintf( $\langle$ format $\rangle,<$ list $\rangle)$ —string formatting.

- \$choice(<list>)—random choice of an element from the list.

The evaluation of such expressions is recursive and allows for some complexity. However, there are limitations in place that prevent abuse of this feature. As an example, \$eval() statements prohibit such things as loops and import statements that would allow the user to write an entire program within an expression. There is also a limit on the number of recursions in order to prevent closed loops in recursive statements.

\subsection{IceProd Server}

The iceprod-server package is comprised of four daemons and their respective libraries:

1. soaptray ${ }^{1}$ an HTTP server that receives client XML-RPC requests for scheduling jobs and steering information which then uploaded to the database.

2. sоарqиеие- a daemon that queries the database for available tasks to be submitted to a particular cluster or grid. This daemon is also responsible for submitting jobs to the cluster or grid through a set of plugin classes.

3. soapmon-a monitoring HTTP server that receives XML-RPC updates from jobs during execution and performs status updates to the database.

4. soapdh-a data handling/garbage collection daemon that removes temporary files and performs any postprocessing tasks.

There are two modes of operation. The first is an unmonitored mode in which jobs are simply sent to the queue of a particular system. This mode provides a tool for scheduling jobs that don't need to be recorded and does not require a database. In the second mode, all parameters are stored in a database that also tracks the progress of each job. The soapqueue daemon running at each of the participating sites periodically queries the database to check if any tasks have been assigned to it. It then downloads the steering configuration and submits a given number of jobs to the cluster or grid where it is running. The number of jobs that IceProd maintains in the queue at each site can be configured individually according to the specifics of each cluster, including the

\footnotetext{
${ }^{1}$ The prefix soap is used for historical reasons. The original implementation of IceProd relied on SOAP for remote procedure calls. This was replaced by XML-RPC which has better support in Python.
}

size of the cluster and local queuing policies. Figure 3 is a graphical representation that describes the interrelation of these daemons. The state diagram in Figure 4 illustrates the role of the daemons in dataset submission while Figure 5 illustrates the flow of information through the various protocols.

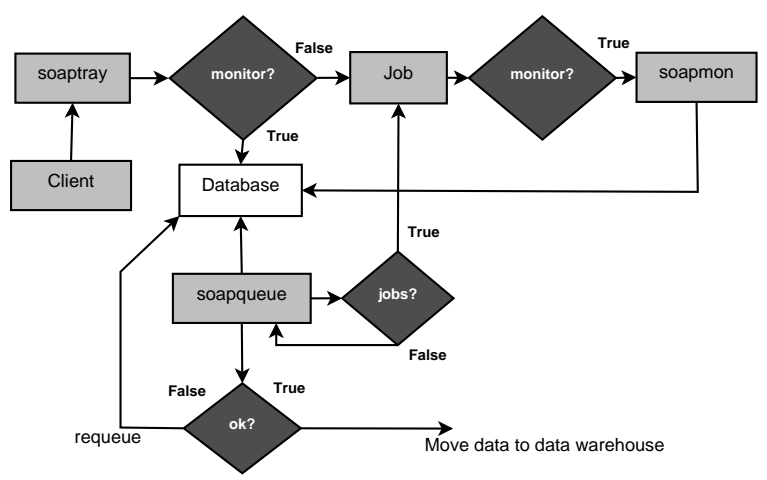

Figure 4: State diagram of queuing algorithm. The iceprod-client sends requests to the soaptray server which then loads the information to the database (in production mode) or directly submits jobs to the cluster (in unmonitored mode). The soарquеие daemons periodically query the database for pending requests and handle job submission in the local cluster.

\subsubsection{IceProd Server Plugins}

In order to abstract the process of job submission from the framework for the various types of systems, IceProd defines a Grid base class that provides an interface for queuing jobs. The Grid base class interface includes a set of methods for queuing and removing jobs, performing status checks, and setting attributes such as job priority and maximum allowed wall time and job requirements such as disk space and memory usage. The set of methods defined by this base class include but are not limited to:

- WriteConfig: write protocol-specific submission scripts (i.e., a JDL job description file in the case of CREAM or gLite or a shell script with the appropriate PBS/SGE headers).

- Submit: submit jobs and record the job ID in the local queue.

- CheckJobStatus: query job status from the queue.

- Remove: cancel/abort a job.

- CleanQ: remove any orphan jobs that might be left in the queue. 


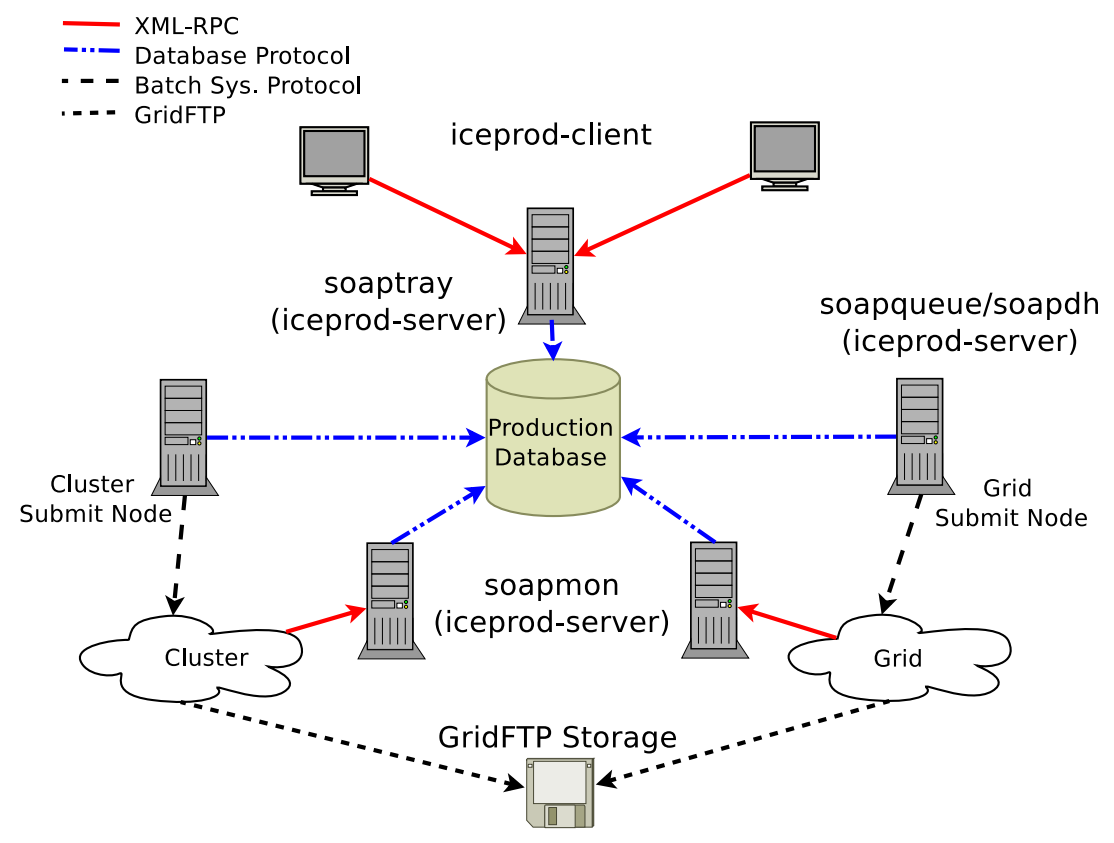

Figure 3: Network diagram of IceProd system. The IceProd clients and JEPs communicate with iceprod-server modules via XML-RPC. Database calls are restricted to iceprod-server modules. Queueing daemons called soapqueue are installed at each site and periodically query the database for pending job requests. The soapmon server receives monitoring update from the jobs. An instance of soapdh handles garbage collection and any post processing tasks after job completion.

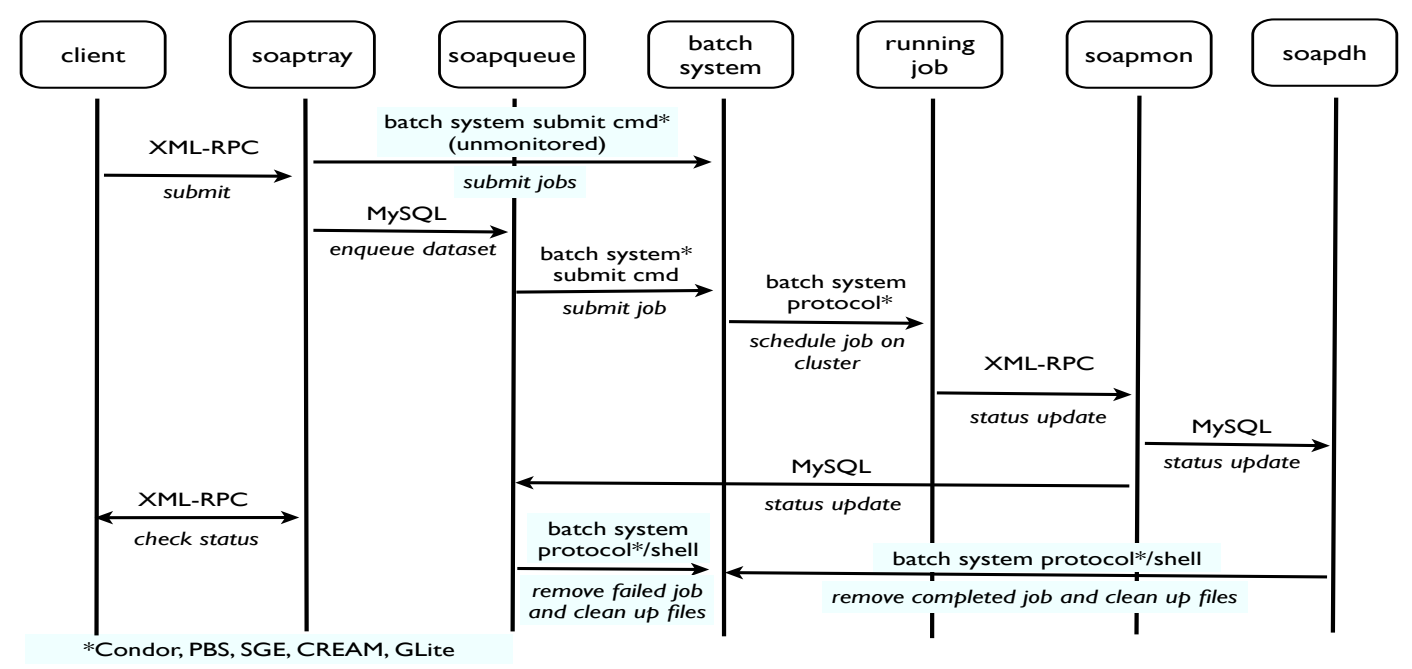

Figure 5: Data flow for job submission, monitoring and removal. Communication between server instances (labeled "soap*") is handled through a database. Client/server communication and monitoring updates are handled via XML-RPC. Interaction with the grid or cluster is handled through a set of plugin modules and depends on the specifics of the system.

The actual implementation of these methods is done by a set of plugin subclasses that launch the corresponding commands or library calls, as the case may be. In the case of PBS and SGE, most of these methods result in the appropriate system calls to qsub, qstat, qdel, etc. For other systems, these can be direct library calls through a Python API. IceProd contains a growing library of plugins, including classes for interfacing with batch systems such as HTCondor, PBS and SGE as well as grid systems like Globus, gLite, EDG, CREAM and ARC. In 
addition, one can easily implement user-defined plugins for any new type of system that is not included in this list.

\subsection{IceProd Modules}

The iceprod-modules package is a collection of configurable modules with a common interface. These represent the atomic tasks to be performed as part of the job. They are derived from a base class IPModule and provide a standard interface that allows for an arbitrary set of parameters to be configured in the XML document and passed from the IceProd framework. In turn, the module returns a set of statistics in the form of a string-to-float dictionary back to the framework so that it can be recorded in the database and displayed on the monitoring web interface. By default, the base class will report the module's CPU usage, but the user can define any set of values to be reported, such as number of events that pass a given processing filter. IceProd also includes a library of predefined modules for performing common tasks such as file transfers through GridFTP, tarball manipulation, etc.

\subsection{External IceProd Modules}

Included in the library of predefined modules is a special module that has two parameters: class and URL. The first is a string that defines the name of an external IceProd module and the second specifies a URL for a (preferably version-controlled) repository where the external module code can be found. Any other parameters passed to this module are assumed to belong to the referred external module and will be ignored. This allows for the use of user-defined modules without the need to install them at each IceProd site. External modules share the same interface as any other IceProd module. External modules are retrieved and cached by the server at the time of submission. These modules are then included as file dependencies for the jobs, thus preventing the need for jobs to directly access the file code repository. Additional precautions, such as enforcing the use of secure protocols for URLs, must be taken to avoid security risks.

\subsection{IceProd Client}

The iceprod-client package contains two applications for interacting with the server and submitting datasets. One is a PyGTK-based GUI (see Figure 6) and the other is a text-based application that can run as a commandline executable or as an interactive shell. Both of these applications allow the user to download, edit, and submit steering configuration files as well as control datasets running on the IceProd-controlled grid. The graphical interface includes drag and drop features for moving modules around and provides the user with a list of valid parameters for known modules. Information about parameters for external modules is not included since these are not known a priori. The interactive shell also allows the user to perform grid management tasks such as starting and stopping a remote server and adding and removing production sites participating in the processing of a dataset. The user can also perform job-specific actions such as suspension and resetting of jobs.

\subsection{Database}

At the time of this writing, the current implementation of IceProd works exclusively with a MySQL database, but all database calls are handled by a database module that abstracts queries from the framework and could be easily replaced by a different relational database. This section describes the relational structure of the IceProd database.

Each dataset is defined by a set of modules and parameters that operate on separate data (single process, multiple data). At the top level of the database structure is the dataset table. The dataset ID is the unique identifier for each dataset, though it is possible to assign a mnemonic string alias. The tables in the IceProd database are logically divided into two distinct classes that could in principle be entirely different databases. The first describes a steering file or dataset configuration (items 1-6 and 9 in the list below) and the second is a job-monitoring database (items 7 and 8). The most important tables are described below.

1. dataset: contains a unique identifier as well as attributes to describe and categorize the dataset, including a textual description.

2. steering-parameter: describes general global variables that can be referenced from any module.

3. meta-project: describes a software environment including libraries and executables.

4. tray: describes a grouping of modules that will execute given the same software environment or metaproject.

5. module: specifies an instance of an IceProd Module class.

6. cparameter: contains all the configured parameters associated with a module.

7. job: describes each job in the queue related to a dataset, including the state and host where the job is executed. 


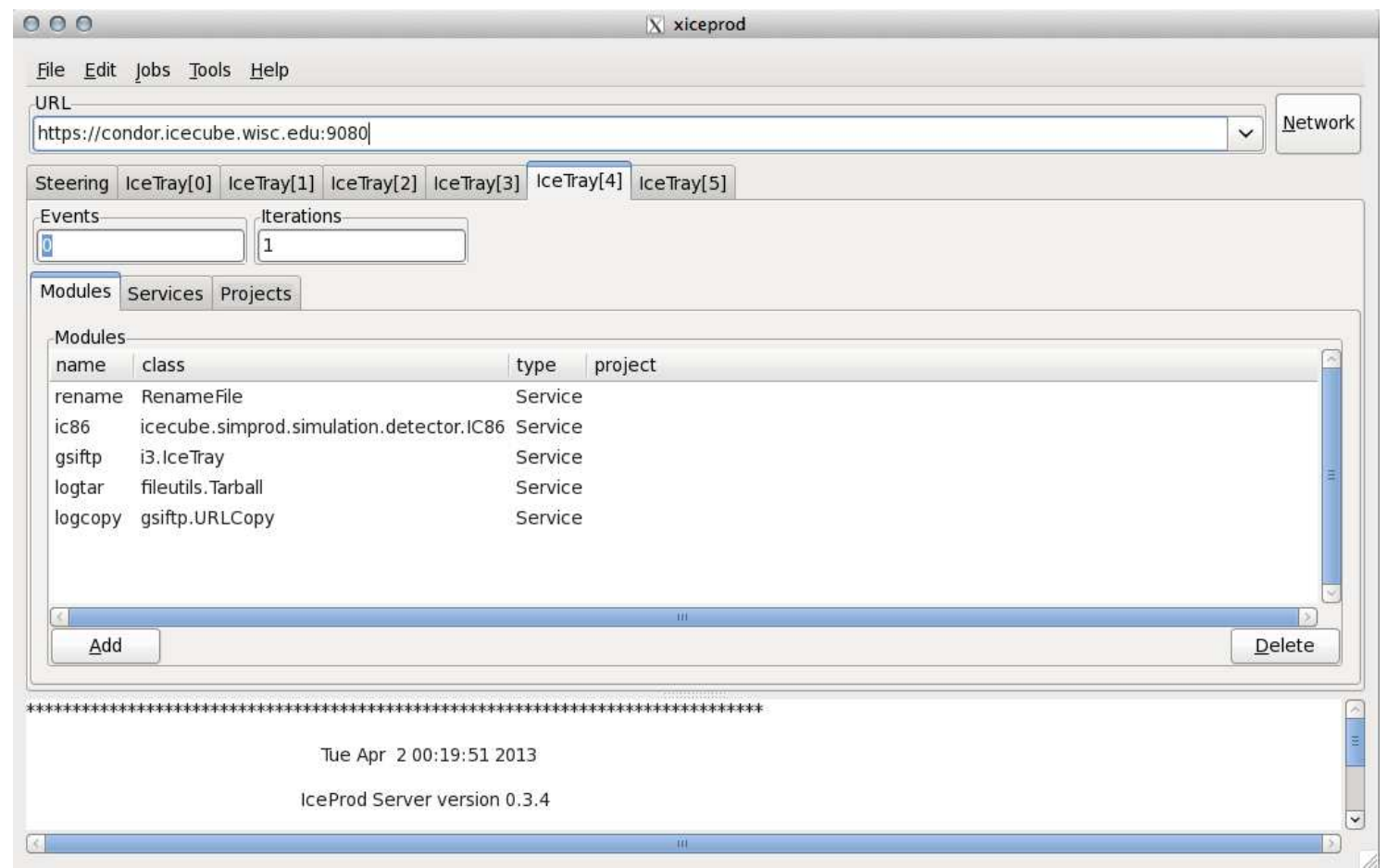

Figure 6: The iceprod-client uses pyGtk and provides a graphical user interface to IceProd. It is both a graphical editor of XML steering files and an XML-RPC client for dataset submission.

8. task: keeps track of the state of a task in a way similar to what is done in the jobs table. A task represents a subprocess for a job in a process workflow. More details on this will be provided in Section 4.

9. task-rel: describes the hierarchical relationship between tasks.

\subsection{Monitoring}

The status updates and statistics are reported by the JEP via XML-RPC to soapmon and stored in the database, and provide useful information for monitoring the progress of processing datasets and for detecting errors. The updates include status changes and information about the execution host as well as job statistics. This is a multi-threaded server that can run as a standalone daemon or as a CGI script within a more robust web server. The data collected from each job are made available for analysis, and patterns can be detected with the aid of visualization tools as described in the following section.

\subsubsection{Web Interface}

The current web interface for IceProd was designed to work independently of the IceProd framework but utilizes the same database. It is written in PHP and makes use of the CodeIgniter framework [10]. Each of the simulation and data-processing web-monitoring tools provide different views, which include, from top level downward:

- general view: displays all datasets filtered by status, type, grid, etc.

- grid view: shows all datasets running on a particular site.

- dataset view: displays all jobs and accompanying statistics for a given dataset, including every site that it is running on.

- job view: shows each individual job, including the status, job statistics, execution host, and possible errors.

There are some additional views that are applicable only to the processing of real IceCube detector data:

- calendar view: displays a calendar with a color coding that indicates the status of jobs associated with data taken on a particular date. 


\begin{tabular}{|c|c|c|c|c|c|c|c|c|c|}
\hline Production & \multicolumn{4}{|c|}{ Any Metaproject } & Any Generator & $\neq$ Any Grid & \multirow{2}{*}{\multicolumn{2}{|c|}{$\theta$}} & \multirow[t]{2}{*}{ Apply Filters } \\
\hline Configuration Files & \multicolumn{6}{|c|}{\begin{tabular}{|lll} 
Dataset 9255 & PROCESSING
\end{tabular}} & & & \\
\hline Datasets & \multicolumn{8}{|c|}{ (1) Suspend } & 8 Resume \\
\hline Wiki & \multirow{2}{*}{\multicolumn{6}{|c|}{ Page: 1234 > }} & \multirow{2}{*}{\multicolumn{3}{|c|}{ Results $1-100$ of $\mathbf{3 2 8}}}$. \\
\hline Jobs & & & & & & & & & \\
\hline Job Queues & $\square$ & ID & Status & Metaproject & Generator & Grid & Host & Errors & Progress \\
\hline Graphs & $\square$ & 9255.60794 & PROCESSING & $\begin{array}{l}\text { simulation } \\
\text { releases.V03-02-01 }\end{array}$ & CORSIKA-in-ice & IceProdDAG.CA & glow-co66 & $\begin{array}{l}\text { o fails } \\
\text { o evicts }\end{array}$ & $57.14 \%$ \\
\hline Grids & $\square$ & 9255.61987 & PROCESSING & $\begin{array}{l}\text { simulation } \\
\text { releases.V03-02-01 }\end{array}$ & CORSIKA-in-ice & IceProdDAG.CA & gzkgooocoog & $\begin{array}{l}\text { o fails } \\
\text { o evicts }\end{array}$ & $71.43 \%$ \\
\hline Search & $\square$ & 9255.61988 & PROCESSING & $\begin{array}{l}\text { simulation } \\
\text { releases.Vo3-02-01 }\end{array}$ & CORSIKA-in-ice & IceProdDAG.CA & e147 & $\begin{array}{l}\text { o fails } \\
\text { o evicts }\end{array}$ & $71.43 \%$ \\
\hline Tickets & $\square$ & 9255.61989 & PROCESSING & $\begin{array}{l}\text { simulation } \\
\text { releases.V03-02-01 }\end{array}$ & CORSIKA-in-ice & IceProdDAG.CA & gzk9ooocoog & $\begin{array}{l}\text { o fails } \\
\text { o evicts }\end{array}$ & $85.71 \%$ \\
\hline Monitors & $\square$ & 9255.61991 & PROCESSING & $\begin{array}{l}\text { simulation } \\
\text { releases.V03-02-01 }\end{array}$ & CORSIKA-in-ice & IceProdDAG.CA & e149 & $\begin{array}{l}\text { o fails } \\
\text { o evicts }\end{array}$ & $85.71 \%$ \\
\hline UW Ganglia Monitor & $\square$ & 9255.62008 & PROCESSING & simulation & CORSIKA-in-ice & IceProdDAG.CA & gzk9ooocoog & o fails & $=$ \\
\hline UW Job Queue & & & & releases.Vo3-02-01 & & & Bangoroucoug & o evicts & $71.43 \%$ \\
\hline UW GLOW Monitor & $\square$ & 9255.62021 & PROCESSING & $\begin{array}{l}\text { simulation } \\
\text { releases.V03-02-01 }\end{array}$ & CORSIKA-in-ice & IceProdDAG.CA & gzk9ooocoo9 & $\begin{array}{l}1 \text { fails } \\
0 \text { evicts }\end{array}$ & $85.71 \%$ \\
\hline
\end{tabular}

Figure 7: A screen capture of the web interface that allows the monitoring of ongoing jobs and datasets. The monitoring web interface has a number of views with different levels of detail. The view shown displays the job progress for active jobs within a dataset. The web interface provides authenticated users with buttons to control datasets and individual jobs.

- day view: shows the status of jobs associated with a given calendar day of data taking.

- run view: displays the status of jobs associated with a particular detector run.

The web interface also provides the functionality to control jobs and datasets by authenticated users. This is done by sending commands to the soaptray daemon using the XML-RPC protocol. Other features of the interface include graphs displaying completion rates, errors and number of jobs in various states. Figure 7 shows a screen capture of one of a number of views from the web interface.

\subsubsection{Statistical Data}

One aspect of IceProd that is not found in most grid middleware is the built-in collection of user-defined statistical data. Each IPModule instance is passed a stringto-float dictionary to which the JEP can add entries or increment a given value. IceProd collects these data in the central database and displays them on the monitoring page. Statistics are reported individually for each job and collectively for the whole dataset as a sum, average and standard deviation. The typical types of information collected on IceCube jobs include CPU usage, number of events meeting predefined physics criteria, and number of calls to a particular module.

\subsection{Security and Data Integrity}

When dealing with network applications, one must always be concerned with security and data integrity in order to avoid compromising privacy and the validity of scientific results. Some effort has been made to minimize security risks in the design and implementation of IceProd. This section will summarize the most significant of these. Figure 3 shows the various types of network communication between the client, server, and worker node.

\subsubsection{Authentication}

Authentication in IceProd can be handled in two ways: IceProd can authenticate dataset submission against an LDAP server or, if one is not available, authentication is handled by means of direct database authentication. LDAP authentication allows the IceProd administrator to restrict usage to individual users that are responsible for job submissions and are accountable for improper use so direct database authentication should be disabled whenever LDAP is available. This setup also precludes the need to distribute database passwords and thus prevents users from being able to directly query the database via a MySQL client.

When dealing with databases, one also needs to be concerned about allowing direct access to the database and passing login credentials to jobs running on remote sites. For this reason, all monitoring calls are done via 
XML-RPC, and the only direct queries are performed by the server, which typically operates behind a firewall on a trusted system. The current web interface does make direct queries to the database; a dedicated readonly account is used for this purpose.

\subsubsection{Encryption}

Both soaptray and soapmon can be configured to use SSL certificates in order to encrypt all data communication between client and server. The encryption is done by the HTTPS server with either a self-signed certificate or, preferably, with a certificate signed by a trusted Certificate Authority (CA). This is recommended for clientserver communication for soaptray but is generally not considered necessary for monitoring information sent to soapmon by the JEP as this is not considered sensitive enough to justify the additional system CPU resources required for encryption.

\subsubsection{Data Integrity}

In order to guarantee data integrity, an MD5 checksum or digest is generated for each file that is transmitted. This information is stored in the database and is checked against the file after transfer. IceProd data transfers support several protocols, but the preference is to rely primarily on GridFTP, which makes use of GSI authentication [11, 12].

An additional security measure is the use of a temporary passkey that is assigned to each job at the time of submission. This passkey is used for authenticating communication between the job and the monitoring server and is only valid during the duration of the job. If the job is reset, this passkey will be changed before a new job is submitted. This prevents stale jobs that might be left running from making monitoring updates after the job has been reassigned.

\section{Intrajob Parallelism}

As described in Section 3.1.2, a single IceProd job consists of a number of trays and modules that execute different parts of the job, for example, a simulation chain. These trays and modules describe a workflow with a set of interdependencies, where the output from some modules and trays is used as input to others. Initial versions of IceProd ran jobs solely as monolithic scripts that executed these modules serially on a single machine. This approach was not very efficient because it did not take advantage of the workflow structure implicit in the job description.

To address this issue, IceProd includes a representation of a job as a directed, acyclic graph (DAG) of tasks.
Jobs are recharacterized as groups of arbitrary tasks and modules that are defined by users in a job's XML steering file, and each task can depend on any number of other tasks in the job. This workflow is encoded in a DAG, where each vertex represents a single instance of a task to be executed on a computing node, and edges in the graph indicate dependencies between tasks (see Figures 8 and 9). DAG jobs on the cluster are executed by means of the HTCondor DAGMan which is a workflow manager developed by the HTCondor group at the University of Wisconsin-Madison and included with the HTCondor batch system [13].

For IceCube simulation production, IceProd has utilized the DAG support in two specific cases: improving task-level parallelism and running jobs that utilize graphics processing units (GPUs) for portions of their processing.

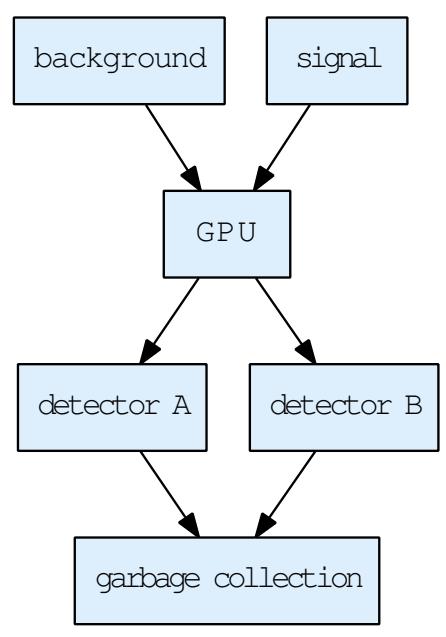

Figure 8: A simple DAG in IceProd. This DAG corresponds to a typical IceCube simulation. The two root vertices require standard computing hardware and produce different types of signal. Their output is then combined and processed on GPUs. The output is then used as input for two different detector simulations.

\subsection{Task-level Parallelism}

In addition to problems caused by coarse-grained requirements specifications, monolithic jobs also underutilize cluster resources. As shown in Figure 8, portions of the workflow within a job are independent; however, if a job is monolithic, these portions will be run serially instead of in parallel. Therefore, although the entire simulation can be parallelized by submitting multiple jobs to different machines, this opportunity for additional parallelism is not exploited by monolithic jobs. 


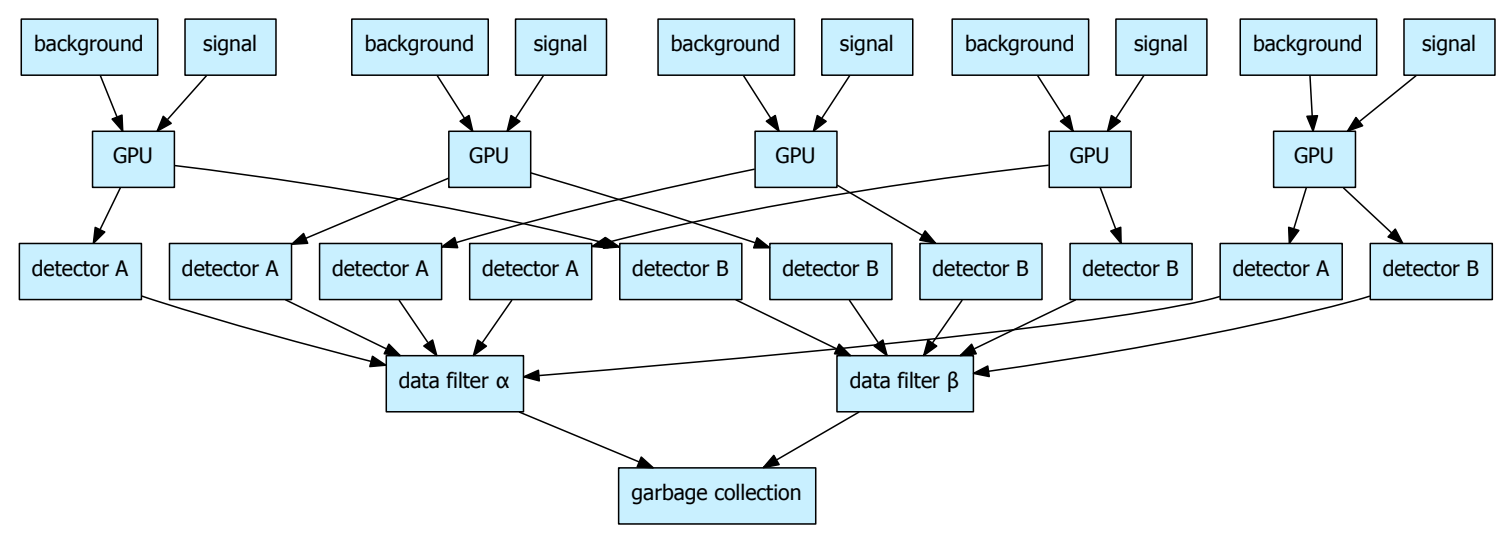

Figure 9: A more complicated DAG in IceProd with multiple inputs and multiple outputs that are eventually merged into a single output. The vertices in the second level run on computing nodes equipped with GPUs.

Support for breaking a job into discrete tasks is now included in the HTCondor IceProd plugin as described above, and similar features have been developed for the PBS and Sun Grid Engine plugins. This enables faster execution of individual jobs by utilizing more computing nodes; however, one limitation of this implementation is that DAG jobs are restricted to a specific type of cluster, and DAG jobs cannot distribute tasks across multiple sites.

\subsection{DAGs Based on System Requirements}

Individual parts of a job may have different system hardware and software requirements. Breaking these up into tasks that run on separate nodes allows for better utilization of resources. The IceCube detector simulation chain is a good example of this scenario in which tasks are distributed across computing nodes with different hardware resources.

Light propagation in the instrumented volume of ice at the South Pole is difficult to model, but recent developments in IceCube's simulation include a much faster approach for simulating direct propagation of photons in the optically complex Antarctic ice $[14,15]$ by using general-purpose GPUs. This new simulation module is much faster than a CPU-based implementation and more accurate than using parametrization tables [16], but the rest of the simulation requires standard CPUs. When executing an IceProd job monolithically, only one set of cluster requirements can be applied when it is submitted to the cluster. Accordingly, if any part of the job requires use of a GPU, the entire monolithic job must be scheduled on a cluster machine with the appropriate hardware.

As of this writing, IceCube has the potential to access 20, 000 CPU cores distributed throughout the world, but only a small number of these nodes are equipped with GPU cards. Because the simulation is primarily CPU bound, the pool of GPU-equipped nodes is not sufficient to run all simulation jobs in an acceptable amount of time. Additionally, this would be an inefficient use of resources, since executing the CPU-oriented portions of monolithic jobs would leave the GPU idle for periods of time. In order to solve this problem, the modular design of the IceCube simulation design is used to divide the CPU- and GPU-oriented portions of jobs into separate tasks in a DAG. Since each task in a DAG is submitted separately to the cluster, their requirements can be specified independently and CPU-oriented tasks can be executed on general-purpose grid nodes while photon propagation tasks can be executed on GPU-enabled machines, as depicted in Figure 9.

\section{Applications}

IceProd's highly configurable nature lets it serve the needs of many different applications, both inside and beyond the IceCube Collaboration.

\subsection{IceCube Simulation Production}

The IceCube simulations are based on a modular software framework called IceTray in which modules are executed in sequential order. Data is passed between modules in the form of a "frame" object. IceCube simulation modules represent different steps in the generation and propagation of particles, in-ice light propagation, signal detection, and simulation of the electronics and data acquisition hardware. These modules are "chained" together in a single IceTray instance but can also be broken into separate instances configured to 
write intermediate data files. This allows for breaking up the simulation chain into multiple IceProd tasks in order to optimize the use of resources as described in Section 4.

For IceCube, Monte Carlo simulations are the most computationally intensive task, which is dominated by the production of background cosmic-ray showers (see Table 2). A typical Monte Carlo simulation lasts on the order of 8 hours but corresponds to only four seconds of detector livetime. In order to generate sufficient statistics, IceCube simulation production needs to make use of available computing resources which are distributed across the world. Table 3 lists all of the sites that have participated in Monte Carlo production.

Table 3: Sites participating in IceCube Monte Carlo production by country.

\begin{tabular}{l|l|r}
\hline Country & Queue Type & No. of Sites \\
\hline \hline Sweden & ARC & 2 \\
\hline Canada & PBS & 2 \\
\hline Germany & SGE & 1 \\
& PBS & 3 \\
& CREAM & 4 \\
\hline Belgium & PBS & 2 \\
\hline USA & HTCondor & 4 \\
& PBS & 3 \\
& SGE & 4 \\
\hline Japan & HTCondor & 1 \\
\hline
\end{tabular}

\subsection{Off-line Processing of the IceCube Detector Data}

IceProd was designed primarily for managing the production of Monte Carlo simulations for IceCube, but it has also been successfully adopted for managing the processing and reconstruction of experimental data collected by the detector. This data collected by IceCube and previously described in Section 1.1 must undergo multiple steps of processing, including calibration, multiple-event track reconstructions, and sorting into various analysis channels based on predefined criteria. IceProd has proved to be an ideal framework for processing this large volume of data.

For off-line data processing, the existing features in IceProd are used for job submission, monitoring, data transfer, verification, and error handling. However, in contrast to a Monte Carlo production dataset where the number of jobs are defined a priori, a configuration for off-line processing of experimental data initiates with an empty dataset of zero jobs. A separate script is then run over the data in order to map a job to a particular file (or group of files) and to generate MD5 checksums for each input file.
Additional minor modifications were needed in order to support the desired features in off-line processing. In addition to the tables described in section 3.6, a run table was created to keep records of runs and dates associated with each file and unique to the data storage structure. All data collected during a season (or a one year cycle) are processed as a single IceProd dataset. This is because, for each IceCube season, all the data collected is processed with the same set of scripts, thus following the SPMD model. A job for such a dataset consists of all the tasks needed to complete the processing of a single data file.

Off-line processing takes advantage of the IceProd built-in system for collecting statistics in order to provide information through web interface about the number of events that pass different quality selection criteria from completed jobs. Troubleshooting and error correction of jobs during processing is also facilitated by IceProd's real-time feedback system accessible through the web interface. The data integrity checks discussed in Section 3.8.3 also provide a convenient way to validate data written to storage and to check for errors during the file transfer task.

\subsection{Off-line Event Reconstruction for the HAWC Gamma-Ray Observatory}

IceProd's scope is not limited to IceCube. Its design is general enough to be used for other applications. The High-Altitude Water Cherenkov (HAWC) Observatory [17] has recently begun using IceProd for its own offline event reconstruction and data transfer [18]. HAWC has two main computing centers, one located at the University of Maryland and one at UNAM in Mexico City. Data is collected from the detector in Mexico and then replicated to UMD. The event reconstruction for HAWC is similar in nature to IceCube's data processing. Unlike IceCube's Monte Carlo production, it is I/O bound and better suited for a local cluster rather than a distributed grid environment. The HAWC Collaboration has made important contributions to the development of IceProd and maintained active collaboration with the development team.

\subsection{Deploying an IceProd Site}

Deployment of an IceProd instance is relatively easy. Installation of the software packages is handled through Python's built-in Module Distribution Utilities package. If the intent is to create a stand-alone instance or to start a new grid, the software distribution also includes scripts that define the MySQL tables required for IceProd. 
After the software is installed, the server needs to be configured through an INI-style file. This configuration file contains three main sections: general queueing options, site-specific system parameters, and job environment. The queueing options are used by the server plugin to help configure submission (e.g. selecting a queue or passing custom directives to the queueing system). System parameters can be used to define the location of a download directory on a shared filesystem or a scratch directory to write temporary files. The job environment can be modified by the server configuration to modify paths appropriately or set other environment variables.

If the type of grid/batch system for the new site is already supported, the IceProd instance can be configured to use an existing server plugin, with the appropriate local queuing options. Otherwise, the server plugin must be written, as described in Section 3.2.1.

\subsection{Extending Functionality}

The ease of adaptation of the framework for the applications discussed in Sections 5.2 and 5.3 illustrates how IceProd can be ported to other projects with minimal customization, which is facilitated by its Python code base.

There are a couple of simple ways in which functionality can be extended: One is through the implementation of additional IceProd Modules as described in Section 3.3. Another is by adding XML-RPC methods to the soapmon module in order to provide a way for jobs to communicate with the server. There are, of course, more intrusive ways of extending functionality, but those require a greater familiarity with the framework.

\section{Performance}

Since its initial deployment in 2006, the IceProd framework has been instrumental in generating Monte Carlo simulations for the IceCube collaboration. The IceCube Monte Carlo production has utilized more than three thousand CPU-core hours distributed between collaborating institutions at an increasing rate and produced nearly two petabytes of data distributed between the two principal storage sites in the U.S. and Germany. Figure 10 shows the relative share of CPU resources contributed towards simulation production. The IceCube IceProd grid has grown from 8 sites to 25 over the years and incorporated new computing resources. Incorporating new sites is trivial since each set of daemons acts as a volunteer that operates opportunistically on a set of job/tasks independent of other sites. There is no

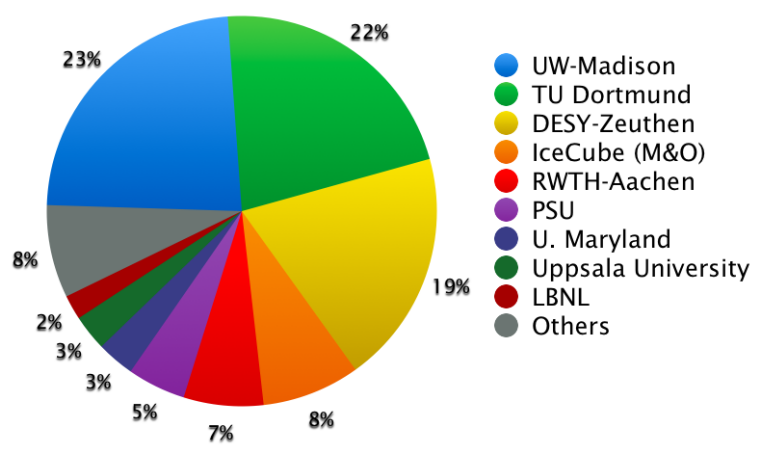

Figure 10: Share of CPU resources contributed by members of the IceCube Collaboration towards simulation production. The relative contributions are integrated over the lifetime of the experiment. The size of the sector reflects both the size of the pool and how long a site has participated in simulation production.

Table 4: IceCube simulation production and off-line processing resource utilization. The production rate has steadily increased since initial deployment. The numbers reflect utilization of owned computing resources and opportunistic ones.

\begin{tabular}{l|r|r}
\hline \hline & Simulation & Off-line \\
\hline Computing centers & 25 & 1 \\
CPU-core time & $\sim 3000 \mathrm{yr}$ & $\sim 160 \mathrm{yr}$ \\
CPU-cores & $\sim 45000$ & 2000 \\
No. of datasets & 2421 & 5 \\
No. of jobs & $1.6 \times 10^{7}$ & $1.5 \times 10^{6}$ \\
No. of tasks & $2.3 \times 10^{7}$ & $1.5 \times 10^{6}$ \\
Data volume & $1.2 \mathrm{~PB}$ & $0.5 \mathrm{~PB}$ \\
\hline
\end{tabular}

central manager that needs to scale with the number of computing sites. The central database is the one component that does need to scale up and can also be a single point of failure. Plans to address this weakness will be discussed in Section 7.

The IceProd framework has also been successfully used for the off-line processing of data collected from the IceCube detector over a 4-year period beginning in the Spring of 2010. This corresponds to 500 terabytes of data and over $3 \times 10^{11}$ event reconstructions. Table 4 summarizes the resources utilized by IceProd for simulation production and off-line processing.

\section{Future Work}

Development of IceProd is an ongoing effort. One important area of current development is the implementation of workflow management capabilities like HTCondor's DAGMan but in a way that is independent of any batch system in order to optimize the use of spe- 
cialized hardware and network topologies by running different job subtasks on different nodes.

Work is also ongoing on a second generation of IceProd designed to be more robust and flexible. The database will be partially distributed to prevent it from being a single point of failure and to better handle higher loads. Caching of files will be more prevalent and easier to implement to optimize bandwidth usage. The JEP will be made more versatile by executing ordinary scripts in addition to modules. Tasks will become a fundamental part of the design rather than an added feature and will therefore be fully supported throughout the framework. Improvements in the new design are based on lessons learned from the first generation IceProd and provide a better foundation on which to continue development.

\section{Conclusions}

IceProd has proven to be very successful for managing IceCube simulation production and data processing across a heterogeneous collection of individual grid sites and batch computing clusters.

With few software dependencies, IceProd can be deployed and administered with little effort. It makes use of existing trusted grid technology and network protocols, which help to minimize security and data integrity concerns that are common to any software that depends heavily on communication through the Internet.

Two important features in the design of this framework are the iceprod-modules and iceprod-server plugins, which allow users to easily extend the functionality of the code. The former provide an interface between the IceProd framework and user scripts and applications. The latter provide an interface that abstracts the details of job submission and management in different grid environments from the framework. IceProd contains a growing library of plugins that support most major grid and batch system protocols.

Though it was originally developed for managing IceCube simulation production, IceProd is general enough for many types of grid applications and there are plans to make it generally available to the scientific community in the near future.

\section{Acknowledgements}

We acknowledge the support from the following agencies: U.S. National Science Foundation-Office of
Polar Programs, U.S. National Science FoundationPhysics Division, University of Wisconsin Alumni Research Foundation, the Grid Laboratory Of Wisconsin (GLOW) grid infrastructure at the University of Wisconsin-Madison, the Open Science Grid (OSG) grid infrastructure; U.S. Department of Energy, and National Energy Research Scientific Computing Center, the Louisiana Optical Network Initiative (LONI) grid computing resources; Natural Sciences and Engineering Research Council of Canada, WestGrid and Compute/Calcul Canada; Swedish Research Council, Swedish Polar Research Secretariat, Swedish National Infrastructure for Computing (SNIC), and Knut and Alice Wallenberg Foundation, Sweden; German Ministry for Education and Research $(\mathrm{BMBF})$, Deutsche Forschungsgemeinschaft (DFG), Helmholtz Alliance for Astroparticle Physics (HAP), Research Department of Plasmas with Complex Interactions (Bochum), Germany; Fund for Scientific Research (FNRS-FWO), FWO Odysseus programme, Flanders Institute to encourage scientific and technological research in industry (IWT), Belgian Federal Science Policy Office (Belspo); University of Oxford, United Kingdom; Marsden Fund, New Zealand; Australian Research Council; Japan Society for Promotion of Science (JSPS); the Swiss National Science Foundation (SNSF), Switzerland; National Research Foundation of Korea (NRF); Danish National Research Foundation, Denmark (DNRF) The authors would like to also thank T. Weisgarber from the HAWC collaboration for his contributions to IceProd development.

\section{Appendix}

The following is a comprehensive list of sites participating in IceCube Monte Carlo production: Uppsala University (SweGrid), Stockholm University (SweGrid), University of Alberta (WestGrid), TU Dortmund (PHiDO, LIDO), Ruhr-Uni Bochum (LiDO), University of Mainz, Université Libre de Bruxelles/Vrije Universiteit Brussel, Universiteit Gent (Trillian) Southern University (LONI), Pennsylvania State University (LIONX), University of Wisconsin (CHTC, GLOW, NPX4), Open Science Grid, RWTH Aachen University (EGI), Universität Dortmund (EGI), Deutsches Elektronen-Synchrotron (EGI, DESY), Universität Wuppertal (EGI), University of Delaware, Lawrence Berkeley National Laboratory (PDSF, Dirac, Carver), University of Maryland. 


\section{References}

[1] F. Halzen, IceCube A Kilometer-Scale Neutrino Observatory at the South Pole, IAU XXV General Assembly, ASP Conference Series 13 (2003) 13-16.

[2] M. G. Aartsen, et al., Search for Galactic PeV gamma rays with the IceCube Neutrino Observatory, Phys. Rev. D 87 (2013) 62002.

[3] F. Halzen, S. R. Klein, IceCube: An Instrument for Neutrino Astronomy, Invited Review Article: Rev. Sci. Inst. 81 (2010) 081101.

[4] D. Protopopescu, K. Schwarz, PANDA Grid-a Tool for Physics, J. Phys.: Conf. Ser. 331 (2011) 072028.

[5] P. Buncic, A. Peters, P. Saiz, The AliEn system, status and perspectives, eConf C0303241 (2003) MOAT004. arXiv:cs/0306067.

[6] R. Brun, F. Rademakers, ROOT - An Object Oriented Data Analysis Framework, Nuclear Inst. and Meth. in Phys. Res., A 389 (1997) 81-86.

[7] A. Pavlo, P. Couvares, R. Gietzel, A. Karp, I. D. Alderman, M. Livny, The NMI build and test laboratory: Continuous integration framework for distributed computing software, Proc. USENIX/SAGE Large Installation System Administration Conference (2006) 263-273.

[8] D. Winer, XML/RPC Specification, http://www.xmlrpc. com/spec (1999).

[9] T. DeYoung, IceTray: A software framework for IceCube, Int. Conf. on Comp. in High-Energy Phys. and Nucl. Phys. (CHEP2004) (2005) 463-466.

[10] R. Ellis, the ExpressionEngine Development Team, CodeIgniter User Guide, http://codeigniter.com (online manual).

[11] W. Allcock, et al., GridFTP: Protocol extensions to FTP for the Grid, http://www.ggf.org/documents/GWD-R/ GFD-R.020.pdf (April 2003).

[12] The Globus Security Team, Globus Toolkit Version 4 Grid Security Infrastructure: A Standards Perspective (2005).

[13] P. Couvares, T. Kosar, A. Roy, J. Weber, K. Wenger, Workflow Management in Condor, In Workflows for e-Science part III (2007) 357-375.

[14] M. G. Aartsen, et al., Measurement of South Pole ice transparency with the IceCube LED calibration system, Nuclear Inst. and Meth. in Phys. Res., A A711 (2013) 73-89.

[15] D. Chirkin, Study of South Pole ice transparency with IceCube flashers, Proc. International Cosmic Ray Conference 4 (2011) 161.

[16] D. Chirkin, Photon tracking with GPUs in IceCube, Nuclear Inst. and Meth. in Phys. Res., A 725 (2013) 141-143.

[17] A. U. Abeysekara, et al., On the sensitivity of the HAWC observatory to gamma-ray bursts HAWC Collaboration, Astropart. Phys. 35 (2012) 641-650.

[18] T. Weisgarber, Production Reconstruction, HAWC Collaboration Meeting, May, 2013 (Unpublished results). 
Juan Carlos Díaz Vélez holds a M. S. in Computer Science and a B. S. in Physics from Boise State University. At Boise State, he studied theoretical models for low-dimensional electron-hole systems, rapidly-rotating Bose-Einstein condensates (BEC), and quantum-dot cellular automata (QDCA). He is currently a systems programmer for the Wisconsin IceCube Particle and Astrophysics Center at University of Wisconsin-Madison. He is the lead for simulation production in the IceCube Collaboration and the main developer of the IceProd distributed computing framework used in Monte Carlo production and data processing for the IceCube experiment. He is also currently working on the High Altitude Water Cherenkov (HAWC) Gamma-ray Observatory in Mexico.

Díaz Vélez's research interest include cosmic rays including cosmic-ray anisotropy, parallel computation, distributed and grid-computing, and numerical simulations. 
*Author Photograph: J.C. Díaz-Vélez

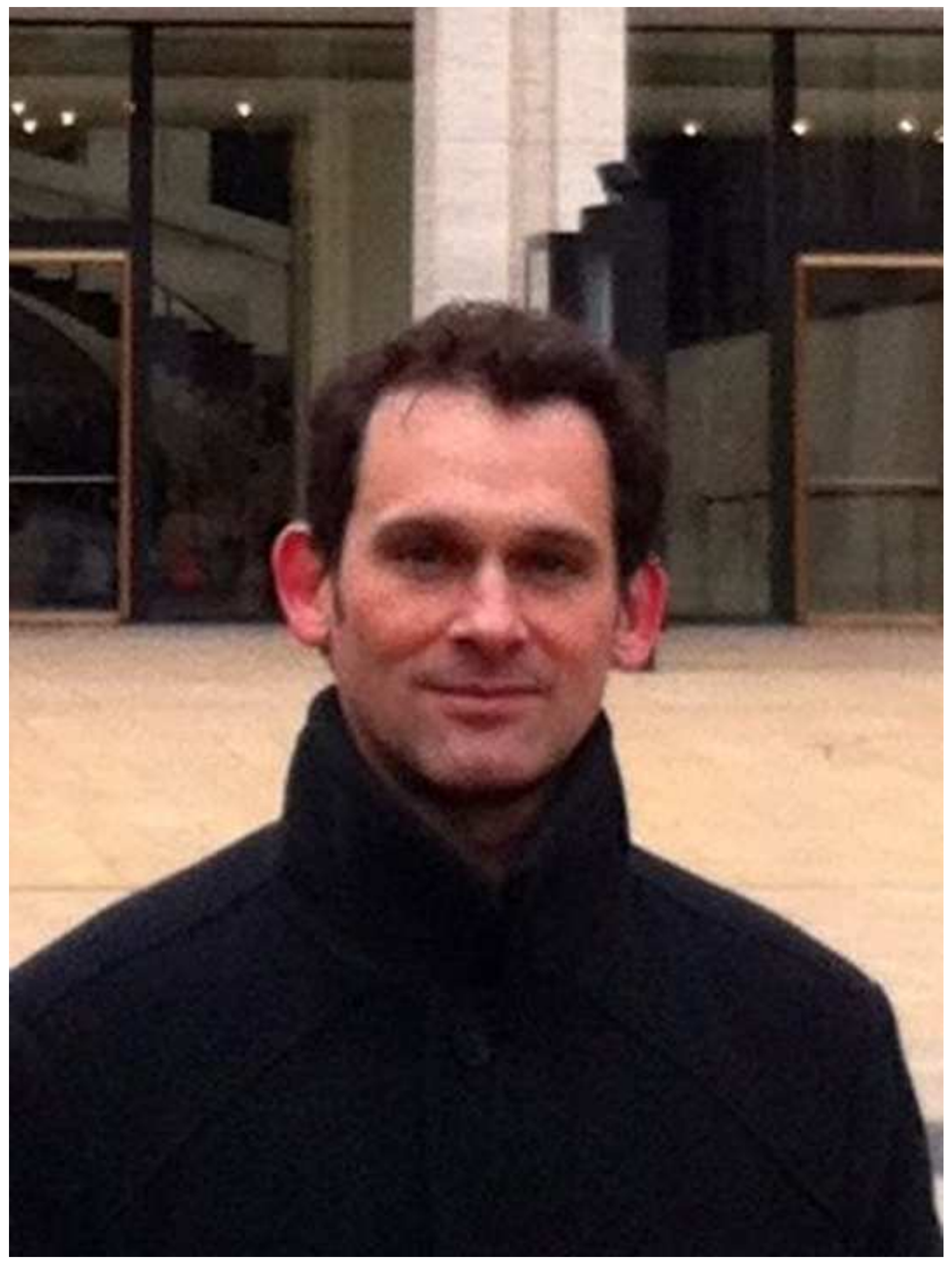


Paolo Desiati is currently an Associate Scientist at the Wisconsin iceCube Particle Astrophysics Center (WIPAC), at the University of Wisconsin - Madison. After working as an undergraduate student in a Cosmic Ray experiment at the Gran Sasso Underground National Laboratory in Italy, Desiati got his $\mathrm{PhD}$ at the University of Rome "La Sapienza', in Roma, Italy, on a Neutrino Telescope project in the Mediterranean. He joined AMANDA, the predecessor of the IceCube Observatory, as a Postdoctoral Fellow at DESY-Zeuthen near Berlin (Germany) and, in 2001 moved to the University of Wisconsin in Madison. Desiati is an active member of the IceCube Collaboration and he is also interested in cosmic ray observations and the study of their propagation in magnetic fields. He collaborates with the Astronomy Department on the effects of the Heliosphere on $\mathrm{TeV}$-energy scale cosmic rays and on the properties of propagation of charged particles perpendicular to magnetic field lines. 
${ }^{*}$ Author Photograph: P. Desiati

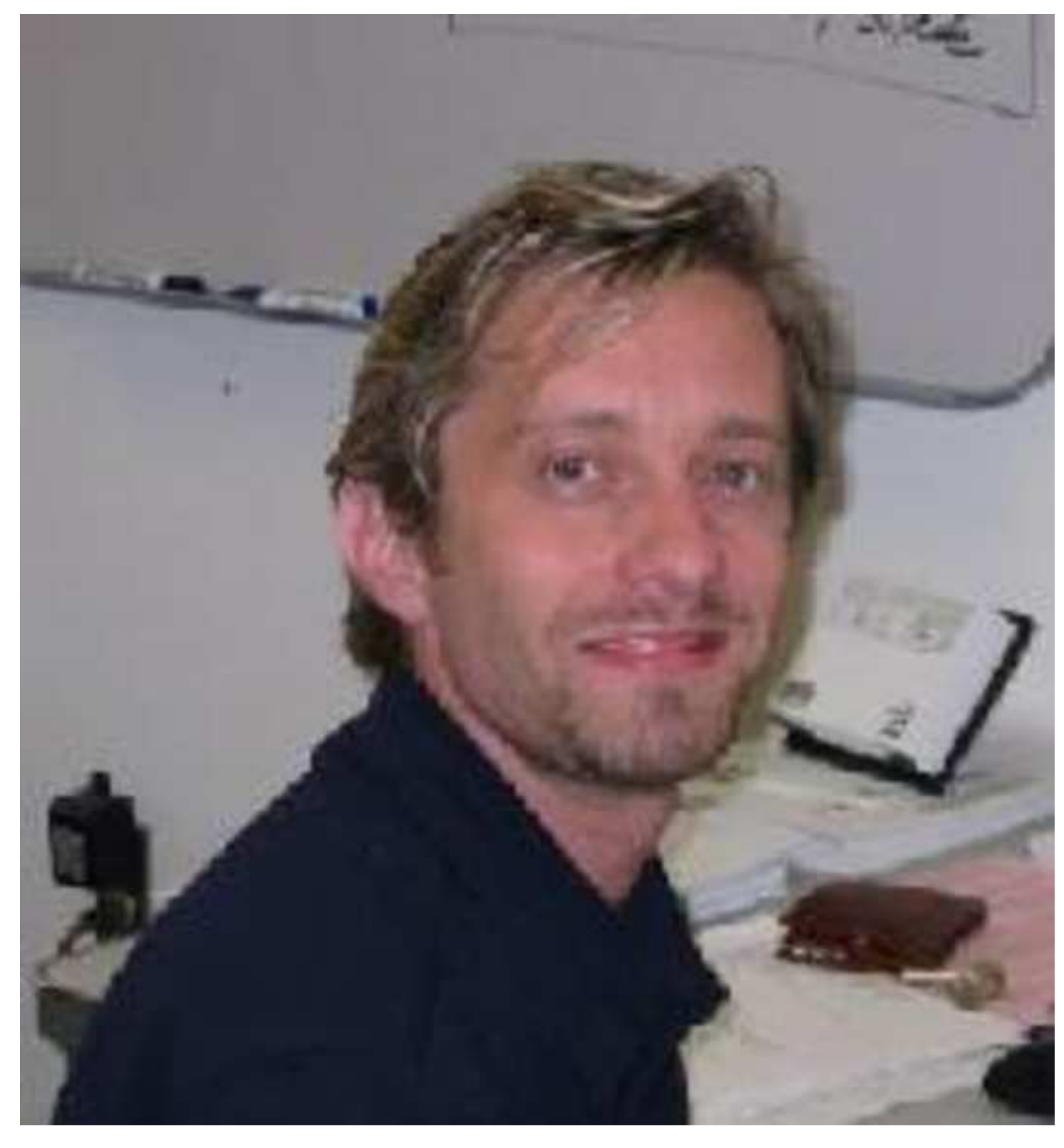

Coph: P. Desiati

^Author Photograph: P. Desiati

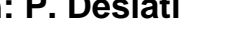

$\sqrt{2}$

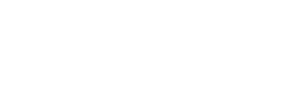

.

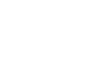

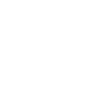

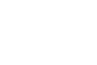


Oladipo O. Fadiran received a B.Eng. in Electrical Engineering from the University of Ilorin, Nigeria. He obtained a M.S. in Electrical Engineering from the University of Cape Town, South Africa and a Ph.D. in Systems Science from Clark Atlanta University (CAU), Atlanta, USA, in 2001 and 2007, respectively. His graduate research included the design of algorithms for analyzing Synthetic Aperture Radar (SAR) and Forward Looking Infrared (FLIR) images. His work, under the supervision of Dr. Michael Inggs, Dr. Lance Kaplan and Dr. Peter Molnar resulted in multiple articles published by the Institute of Electrical and Electronics Engineers (IEEE) and International Society for Optics and Photonics (SPIE), among others. He worked as a research/teaching associate at CAU from 2007-2009. He has been employed as a programmer with the Wisconsin IceCube Particle Astrophysics Center (WIPAC), at the University of Wisconsin, Madison since 2009. He works with a group responsible for processing data from, and the Monte Carlo simulation of the IceCube Neutrino detector. 


\section{Photograph: O. Fadiran}
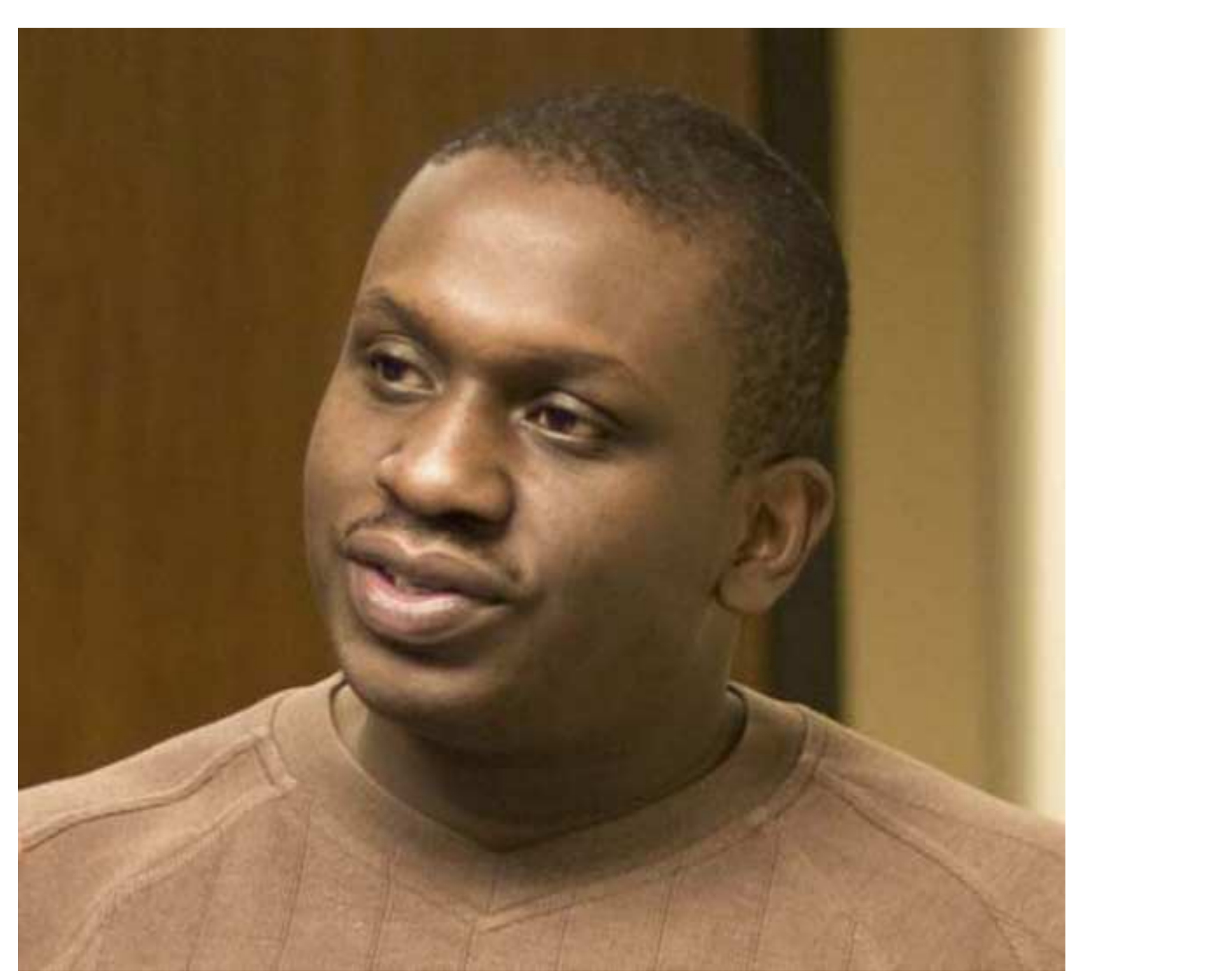

(2)
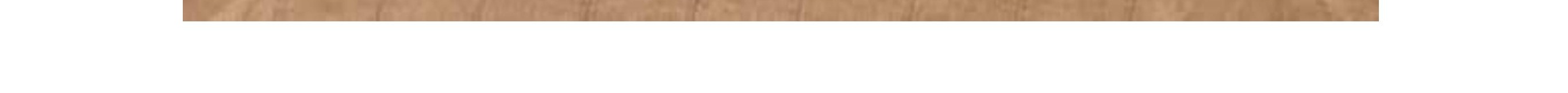
Ian Rae holds a M.S. and B.S. in Computer Science from University of Wisconsin-Madison and is currently a Ph.D. candidate at U.W. lan worked as a research assistant for the IceCube Neutrino Observatory and helped develop the IceProd distributed computing framework. His Ph.D. research has focused on two broad areas: full-text search in relational databases and executing relational queries in a Hadoop environment.

$\mathrm{He}$ is currently working on development of Knot, a relational processing engine built on top of YARN, the next-generation job scheduler in Hadoop 2.0. Knot is a dedicated relational engine that allows relational queries to be formulated in terms of operator trees, with a great deal of flexibility in scheduling and pipelining of operators. By building on top of YARN, Knot maintains good compatibility with Hadoop, but provides a much more natural interface to execute relational operations. 


\section{${ }^{*}$ Author Photograph: I. Rae}
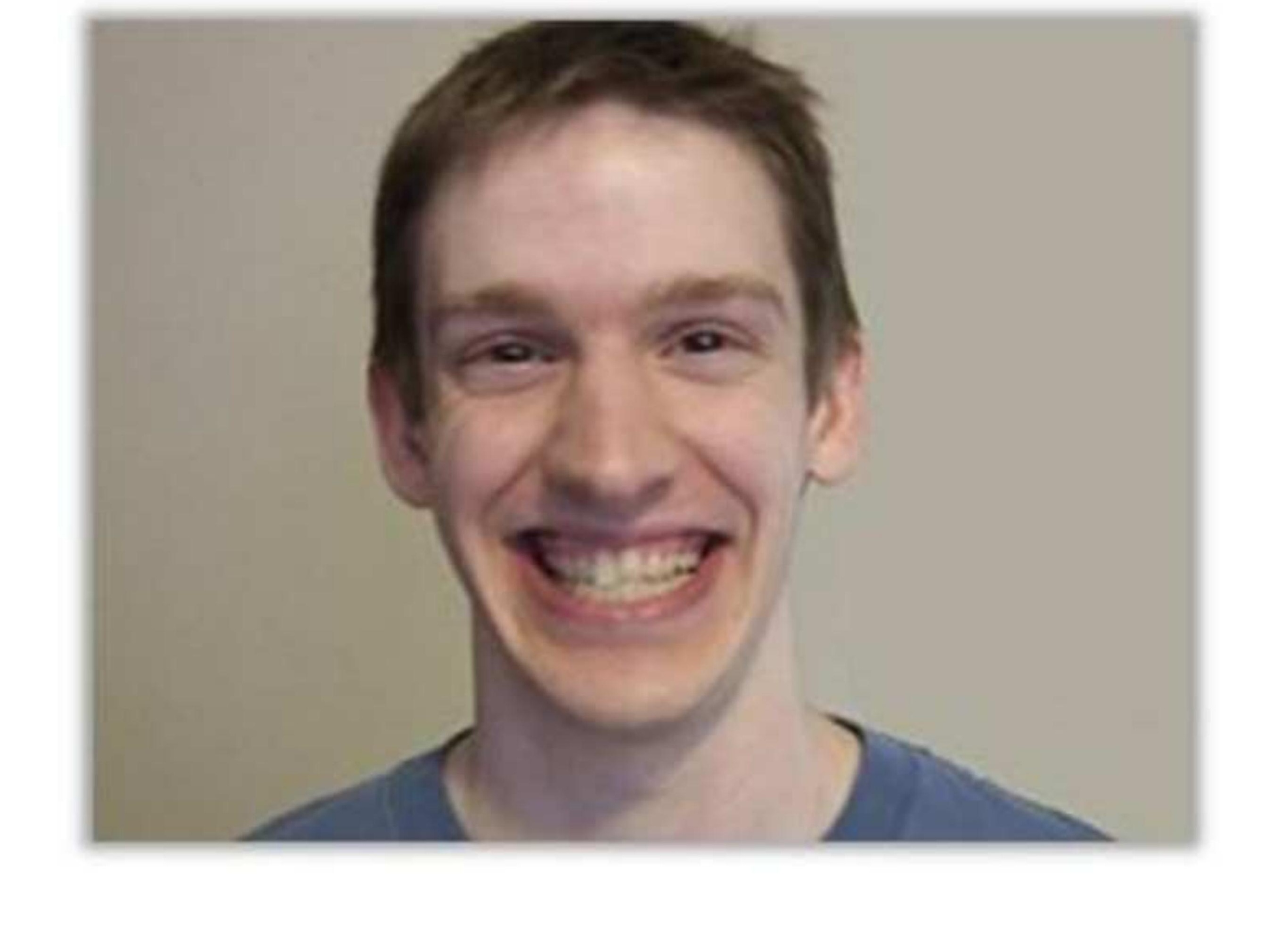

.

\section{Author Photograph: I. Rae}

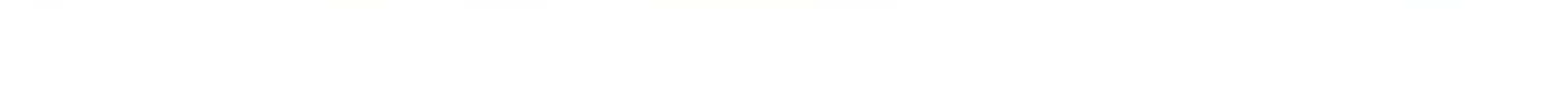


David Schultz is a Systems Programmer at the IceCube Neutrino Observatory at University of Wisconsin-Madison. He received a B.S. degree from Carnegie Mellon University in 2010. His research interests include large-scale data intensive computing, grid middleware, scheduling in distributed systems, and GPGPU computing. 


\section{${ }^{*}$ Author Photograph: D. Schultz}

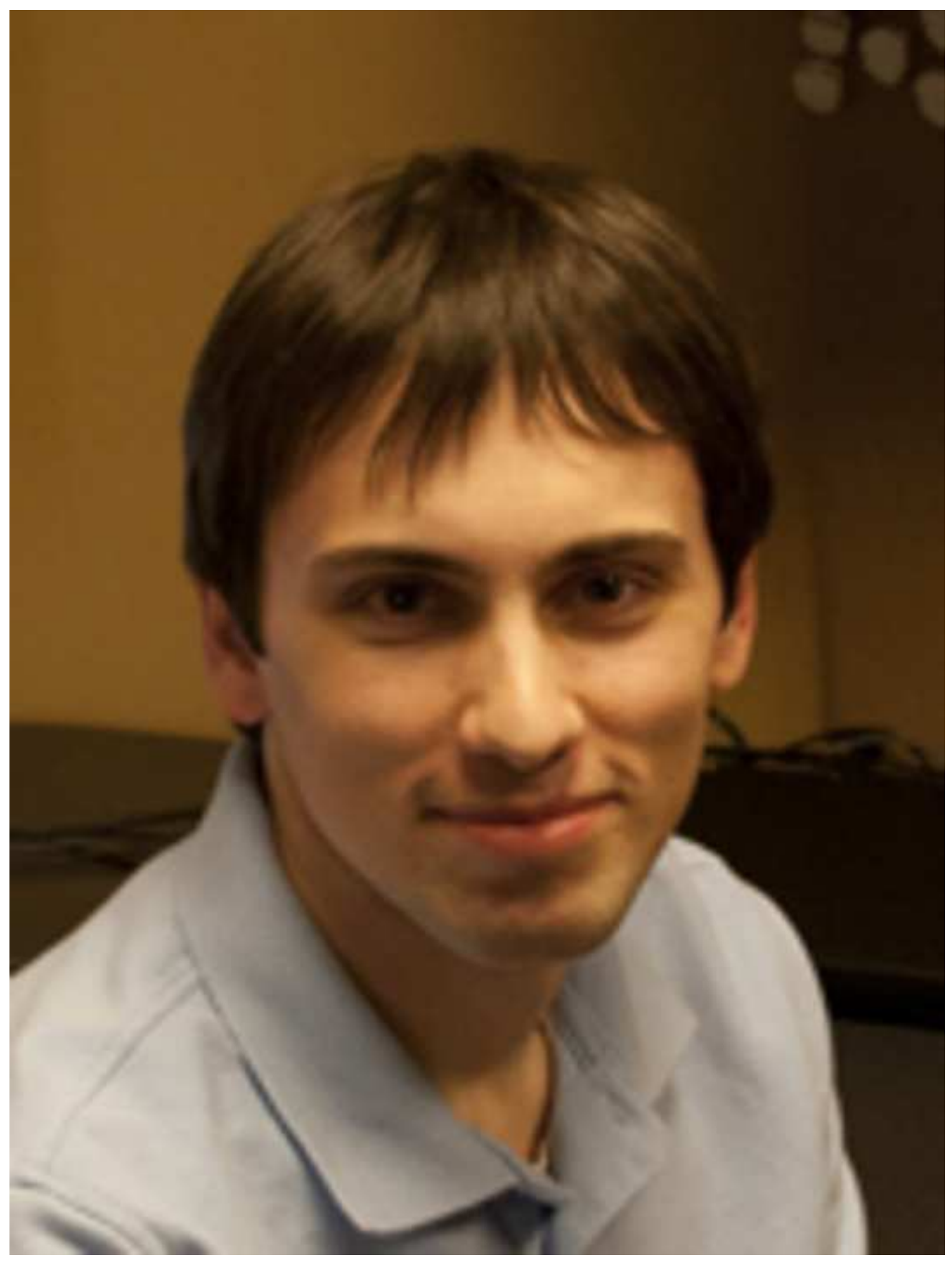

